\title{
Grand-Canonical-like Molecular-Dynamics Simulations by Using an Adaptive-Resolution Technique
}

\begin{abstract}
Han Wang, Carsten Hartmann, Christof Schütte, and Luigi Delle Site*
Institute for Mathematics, Freie Universität Berlin, Arnimallee 6, D-14195 Berlin, Germany

(Received 18 October 2012; revised manuscript received 5 December 2012; published 8 March 2013)

In this work, we provide a detailed theoretical analysis, supported by numerical tests, of the reliability of the adaptive-resolution-simulation (AdResS) technique in sampling the grand-canonical ensemble. We demonstrate that the correct density and radial distribution functions in the hybrid region, where molecules change resolution, are two necessary conditions for considering the atomistic and coarse-grained regions in AdResS to be equivalent to subsystems of a full atomistic system with an accuracy up to the second order with respect to the probability distribution of the system. Moreover, we show that the work done by the thermostat and a thermodynamic force in the transition region is formally equivalent to balancing the chemical potential difference between the different resolutions. From these results follows the main conclusion that the atomistic region exchanges molecules with the coarse-grained region in a grand-canonical fashion with an accuracy up to (at least) second order. Numerical tests, for the relevant case of liquid water at ambient conditions, are carried out to strengthen the conclusions of the theoretical analysis. Finally, in order to show the computational convenience of AdResS as a grand-canonical setup, we compare our method to the insertion particle method in its most efficient computational implementation. This fruitful combination of theoretical principles and numerical evidence makes the adaptive-resolution technique a candidate for a natural, general, and efficient protocol for grand-canonical molecular dynamics for the case of large systems.
\end{abstract}

DOI: 10.1103/PhysRevX.3.011018

\section{INTRODUCTION}

The adaptive-resolution-simulation (AdResS) scheme $[1,2]$ is a method that in a concurrent fashion simulates a molecular system treated with different resolutions in different regions of space. The word "resolution" here refers to the amount of details included in a molecular model: The higher resolution corresponds to a more precise model but at higher computational costs. The advantage is obvious: It keeps track of both the local fine-grained processes in the high-resolution regions and the large-scale behavior, requiring a less demanding computational effort compared to the system that, as a whole, would be described by the highresolution model. The key feature of AdResS is that it allows for an on-the-fly change of resolution when a molecule travels from a high-resolution region to a lowresolution region and vice versa. Moreover, recent research $[3,4]$ has numerically demonstrated that different regions (with different resolutions) reach a thermodynamic equilibrium, as if the whole system were equilibrated under the high-resolution description. Therefore, one is tempted to state that a region with a certain resolution exchanges molecules with the rest of the system in a grand-canonical fashion. This work studies the minimal necessary conditions, such that AdResS performs effective grand-canonical

\footnotetext{
*luigi.dellesite@fu-berlin.de

Published by the American Physical Society under the terms of the Creative Commons Attribution 3.0 License. Further distribution of this work must maintain attribution to the author(s) and the published article's title, journal citation, and DOI.
}

simulations. Moreover, we shed light on the level of accuracy of the sampling in a grand-canonical fashion; it will be shown that the sampling can indeed be considered to be of the grand-canonical type if we accept an accuracy on the probability distribution of the system up to the second order. Although the second order is numerically satisfying, a higher level of accuracy can be systematically reached by requiring higher orders of the probability distribution to match in the transition region.

From the numerical point of view, for systems that are large enough, a (relatively) small subsystem of a full atomistic region is a natural grand-canonical ensemble. For this reason, the approach we use here is that of showing the equivalence between the atomistic region of AdResS and the same region in a full atomistic simulation. Extensive numerical tests, for the relevant case of liquid water at ambient conditions, are presented in order to show that the hypothesis done within the theoretical analysis is justified from the numerical point of view. Beyond our expectations, we find that, in the atomistic region of AdResS, not only can the accuracy be tuned up to the second order, but, without any additional correction, even the three-body correlation function of molecular centers of mass (COM) turns out to be the same as that of a full atomistic simulation. Finally, a further numerical test is carried out: The coarse-grained molecules are substituted with a liquid of spheres that interact via the Weeks-Chandler-Andersen (WCA) potential [5], which has no reference to the atomistic resolution. We show that, also in this case, in the atomistic region, due to the work of the filter of the transition region, an accuracy up to the third order in the probability distribution is achieved. 
The results of this work allow us to make a further step in the possibility of employing the AdResS scheme as a natural, general numerical protocol for truly grand-canonical molecular-dynamics simulations independently from the nature of the particles that act as a reservoir in the coarsegrained region. The word "natural" refers to the fact that, strictly speaking in statistical mechanics, the grandcanonical ensemble is defined in operative terms as a subsystem of an infinitely large system. Of course, in simulation, systems are never infinitely large; however, they can be large enough to numerically satisfy this condition. In this sense, in our approach, molecules are exchanged between the atomistic domain and the coarse-grained reservoir in a straightforward (i.e., natural) dynamical way. The advantage, compared to previous grand-canonical schemes for molecular dynamics, is that the proper exchange of particles requires neither the knowledge or calculation of the chemical potential nor additional expensive insertion or removal of particles [6-11]. We actually compare the computational costs of our method to those of the insertion particle method (IPM) and show that, for dense liquid systems, AdResS is more efficient. The paper is organized as follows: First, we briefly describe the essential principles on which AdResS is based, and then we discuss the approximations under which one can write the probability distribution of the system in terms of the grand-canonical partition function. Next, we show that the work of the thermostat plus that of the thermodynamic force balances the difference in chemical potential; this idea allows us to derive the necessary conditions of the simulation to reach the accuracy in terms of orders of the probability distribution of the system. Finally, the numerical results and the efficiency of the method are discussed.

\section{A BRIEF DESCRIPTION OF THE AdResS SCHEME}

In this work, the higher resolution refers to the atomistic (AT) description of a molecule, while the lower resolution refers to its corresponding coarse-grained (CG) model. (For a pictorial representation of a typical AdResS setup, see Fig. 1 for the case of liquid water.) We assume that the dynamics of the system is subject to the Langevin equation, which is the thermostat that is usually used in the AdResS simulations. We denote the phase-space variable as $\boldsymbol{x}=$ $\left(\boldsymbol{r}_{1}, \ldots, \boldsymbol{r}_{N}, \boldsymbol{v}_{1}, \ldots, \boldsymbol{v}_{N}\right) ; \boldsymbol{r}_{i}$ and $\boldsymbol{v}_{i}$ are the center-of-mass degrees of freedom (DOF) of the molecules. For simplicity, we do not explicitly write the atomistic DOF, but one should keep in mind that the interaction between two molecules with atomistic resolution is given by the sum of all pairwise atomic interactions. Also, for the sake of simplicity, we do not explicitly distinguish between the $\mathrm{COM}$ and the atomistic coordinates, using a generalized formalism, where the coordinates are indicated simply as $\boldsymbol{r}_{i}$; however, the interpretation of such a notation is made clear by the specific context. If the system is conservative, then one obtains the equilibrium density distribution $p(\boldsymbol{x}) \propto e^{-\beta \mathcal{H}(x)}$, where $\beta=1 / k_{B} T$ is the inverse temperature. In this paper, we assume that the kinetic part of the Hamiltonian is decoupled from the configurational part; then, it is usually more convenient to consider the configurational probability distribution, namely, $p\left(\boldsymbol{r}_{1}, \ldots, \boldsymbol{r}_{N}\right)=$ $\int p\left(\boldsymbol{r}_{1}, \ldots, \boldsymbol{r}_{N}, \boldsymbol{v}_{1}, \ldots, \boldsymbol{v}_{N}\right) d \boldsymbol{v}_{1}, \ldots, d \boldsymbol{v}_{N}$. Throughout the paper, when we mention the $i$ th order of a multibody configurational probability distribution, e.g., $p\left(\boldsymbol{r}_{1}, \ldots, \boldsymbol{r}_{N}\right)$, we mean its $i$ th marginal distribution, which is defined by $p^{(i)}\left(\boldsymbol{r}_{1}, \ldots, \boldsymbol{r}_{i}\right)=\int p\left(\boldsymbol{r}_{1}, \ldots, \boldsymbol{r}_{i}, \boldsymbol{r}_{i+1}, \ldots, \boldsymbol{r}_{N}\right) d \boldsymbol{r}_{i+1}, \ldots, d \boldsymbol{r}_{N}$. It is obvious that, whenever we have the $i$ th-order accuracy of a probability distribution, any lower order is automatically accurate. In the AdResS scheme, different resolutions in the system are described by a weighting function $w(\boldsymbol{r})$. Usually, the higher resolution is denoted by $w=1$, while the lower resolution is denoted by $w=0$. Between the higher and lower resolutions, a hybrid region allows a molecule to have both (interpolated) resolutions. The weighting function changes smoothly from 0 to 1 ; one possible form of such a function is

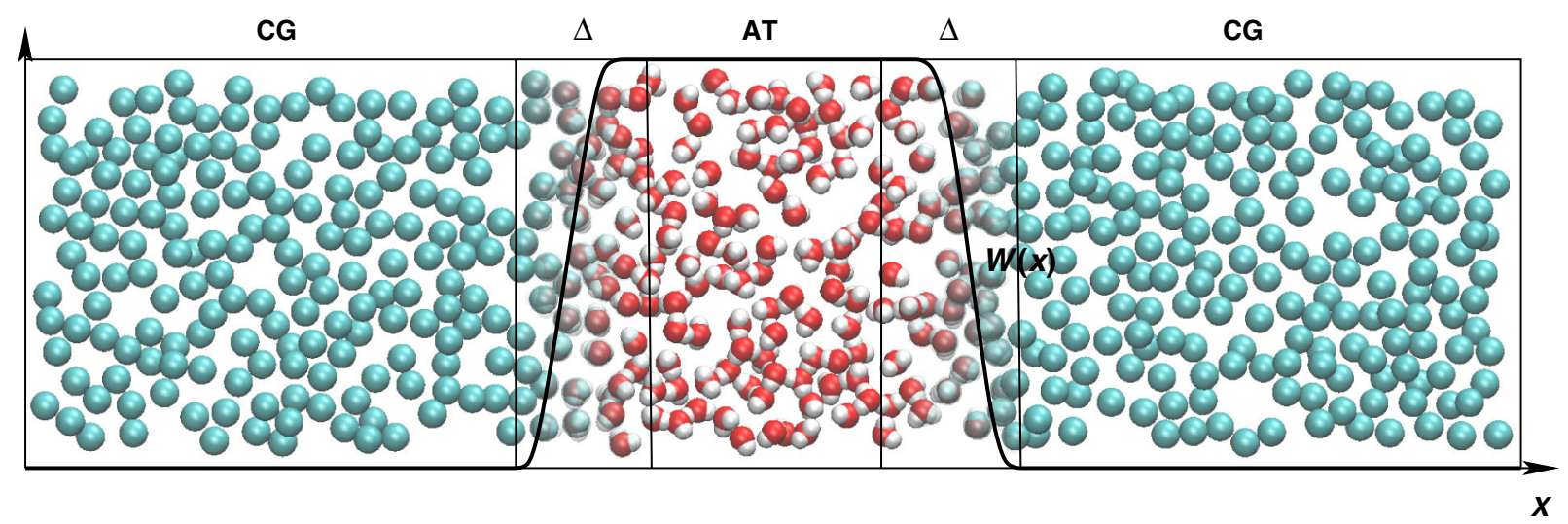

FIG. 1. A schematic representation of liquid water in AdResS. CG indicates the region at coarse-grained resolution, AT that at atomistic resolution, and finally $\Delta$ the transition region where molecules change resolution according to a smooth switching function $w(x)$. 


$$
w(\boldsymbol{r})= \begin{cases}1 & \chi(\boldsymbol{r})<0 \\ 1 & 0<\chi(\boldsymbol{r})<r_{c} \\ \cos ^{2}\left[\frac{\pi}{2\left(d_{\Delta}-r_{c}\right)}\left[\chi(\boldsymbol{r})-r_{c}\right]\right] & r_{c}<\chi(\boldsymbol{r})<d_{\Delta} \\ 0 & d_{\Delta}<\chi(\boldsymbol{r}),\end{cases}
$$

where $\chi(\boldsymbol{r})$ is the distance between the molecule at $\boldsymbol{r}$ and the boundary of the higher-resolution region, $\chi(\boldsymbol{r})<0$ means that the molecule is in the high-resolution region, $d_{\Delta}$ is the thickness of the hybrid region, and $r_{c}$ is the cutoff radius of the atomistic interactions. The intermolecular force is modeled by the following interpolation formula [4],

$\boldsymbol{F}_{i j}=w_{i} w_{j} \boldsymbol{F}_{i j}^{\mathrm{AT}}+\left(1-w_{i} w_{j}\right) \boldsymbol{F}_{i j}^{\mathrm{CG}}+w_{i} w_{j}\left(1-w_{i} w_{j}\right) \boldsymbol{F}_{i j}^{\mathrm{RDF}}$,

where $w_{i}=w\left(\boldsymbol{r}_{i}\right)$ and $w_{j}=w\left(\boldsymbol{r}_{j}\right) . \boldsymbol{F}_{i j}^{\mathrm{AT}}$ and $\boldsymbol{F}_{i j}^{\mathrm{CG}}$ are the intermolecular interactions of the atomistic and coarsegrained resolutions, respectively. $\boldsymbol{F}_{i j}^{\mathrm{RDF}}$ is a force that corrects the COM-COM radial distribution function (RDF) in the transition region $\Delta[4]$. A further one-body force, called thermodynamic force, $\boldsymbol{F}_{\text {th }}$, is applied to ensure the correct thermodynamic equilibrium of the system (for specific details, see Ref. [3]):

$$
\boldsymbol{F}_{i}=\sum_{j} \boldsymbol{F}_{i j}+\boldsymbol{F}_{\mathrm{th}}\left(\boldsymbol{r}_{i}\right)
$$

which is defined by

$$
p_{\mathrm{AT}}+\rho_{0} \int_{\Delta} \boldsymbol{F}_{\mathrm{th}}(\boldsymbol{r}) d \boldsymbol{r}=p_{\mathrm{CG}},
$$

where $p_{\mathrm{AT}}$ is the pressure of the atomistic resolution, $p_{\mathrm{CG}}$ is that of the coarse-grained resolution, and $\rho_{0}$ is the equilibrium number density that corresponds to that of a full atomistic simulation. Such a thermodynamic force has been derived by empirical considerations regarding the equilibrium of open systems and is based on forcing the equality of the grand potential of the atomistic part with the rest of the system. Forcing the equality is then reduced to provide a balancing force to the difference of pressure at the wished uniform density of equilibrium. Regarding this point, one of the main results of this paper is that the thermodynamic force and the thermostat perform work in the transition region that balances the difference in chemical potential between the different regions. As a consequence (equilibrium of the chemical potential), the interpretation of the AdResS simulation as a grand-canonical sampling is enforced even further. A crucial point of this work is the following. The AdResS scheme is not Hamiltonian $[12,13]$; however, under the hypothesis of fixing DOF in the hybrid region for a statistical analysis, the atomistic and coarse-grained regions can be considered (in a good approximation) Hamiltonian. This line of thought gives rise to the basic idea of the present study as it is presented in the next sections. Moreover, Refs. [12,13] also show that a space-dependent interpolation of potentials, which would lead to a direct Hamiltonian approach, is satisfactory neither from the point of view of the physical consistency nor for the computational efficiency in improving in a systematic way the accuracy of the results in the AT region. Instead, improving the accuracy is one of the main results of this work.

\section{THEORETICAL CONSIDERATIONS}

\section{A. The outline of the basic idea}

Here, we denote the degrees of freedom and number of particles in the AT region, the hybrid region $(\Delta)$, and the CG region by $\left(\boldsymbol{x}_{1}, N_{1}\right),\left(\boldsymbol{x}_{2}, N_{2}\right)$, and $\left(\boldsymbol{x}_{3}, N_{3}\right)$, respectively. Therefore, the target is to prove that the atomistic region is subject to the grand-canonical statistics:

$$
p\left(\boldsymbol{x}_{1}, N_{1}\right)=\frac{1}{\mathcal{Q}_{1}} e^{\beta \mu_{\mathrm{AT}} N_{1}-\beta \mathcal{H}_{N_{1}}^{\mathrm{AT}}\left(\boldsymbol{x}_{1}\right)},
$$

where the partition function $\mathcal{Q}_{1}$ is defined by

$$
\mathcal{Q}_{1}=\sum_{N_{1}} \int d \boldsymbol{x}_{1} e^{\beta \mu_{\mathrm{AT}} N_{1}-\beta \mathcal{H}_{N_{1}}^{\mathrm{AT}}\left(x_{1}\right)}
$$

The marginal probability of finding $N_{1}$ molecules in the AT region is

$$
p\left(N_{1}\right)=\int d \boldsymbol{x}_{1} p\left(\boldsymbol{x}_{1}, N_{1}\right)=\frac{1}{\mathcal{Q}_{1}} e^{\beta \mu_{\mathrm{AT}} N_{1}} Q_{N_{1}},
$$

where $Q_{N_{1}}$ is the partition function for a canonical ensemble with $N_{1}$ atomistic molecules:

$$
Q_{N_{1}}=\int d \boldsymbol{x}_{1} e^{-\beta \mathcal{H}_{N_{1}}^{\mathrm{AT}}\left(\boldsymbol{x}_{1}\right)} .
$$

Let us consider $p\left(\boldsymbol{x}_{1}, N_{1}\right)=p\left(\boldsymbol{x}_{1} \mid N_{1}\right) p\left(N_{1}\right)$. Then, from Eqs. (5) and (7), the conditional probability $p\left(x_{1} \mid N_{1}\right)$ for a truly grand-canonical ensemble turns out to be

$$
p\left(\boldsymbol{x}_{1} \mid N_{1}\right)=\frac{1}{Q_{N_{1}}} e^{-\beta \mathcal{H}_{N_{1}}^{\mathrm{AT}}\left(\boldsymbol{x}_{1}\right)} .
$$

The key point of our argumentation is the following: We want to compare the distributions (in the various regions) of the AdResS simulation with the corresponding ones of a full atomistic reference system, which is ideally divided in subregions corresponding to the AT, $\Delta$, and CG regions of the AdResS setup. The first step in our procedure is to fix the number of molecules in the atomistic region and consider the conditional probability $p\left(\boldsymbol{x}_{1} \mid N_{1}\right)$. If the AdResS setup would sample the space in a grand-canonical fashion, then this probability should be the same as the corresponding one of a full atomistic reference system, namely, Eq. (9). Then, if the probability of finding $N_{1}$ molecules in the atomistic region is also the same as the full atomistic reference system, namely, Eq. (7), we can safely state that the atomistic region of AdResS samples configurations in a grand-canonical fashion. In fact, as underlined before, it must be noticed that a subsystem of the full atomistic 
system is a natural grand-canonical ensemble in the thermodynamic limit (see, e.g., Ref. [14]). The thermodynamic limit in our case is intended in such a way that, at a fixed density, the sizes of both the AT and CG regions tend to the infinite, and, at the same time, the ratio between the extension of the AT region and that of the $\mathrm{CG}$ regions goes to zero. As underlined before, in practical terms, in numerical simulation, the system size does not go to infinity; however, it can be large enough so that, within a certain numerical accuracy, the hypothesis of the thermodynamic limit holds.

\section{B. The conditional probability density $p\left(x_{1} \mid N_{1}\right)$}

To prove the above statement about $p\left(\boldsymbol{x}_{1} \mid N_{1}\right)$, we divide it into two parts:

$p\left(\boldsymbol{x}_{1} \mid N_{1}\right)=\sum_{N_{2}} \int p\left(\boldsymbol{x}_{1} \mid N_{1} ; \boldsymbol{x}_{2}, N_{2}\right) p\left(\boldsymbol{x}_{2}, N_{2} \mid N_{1}\right) d \boldsymbol{x}_{2}$,

where $p\left(\boldsymbol{x}_{1} \mid N_{1} ; \boldsymbol{x}_{2}, N_{2}\right)$ is the probability density obtained by fixing the coordinates and number of particles in the region $\Delta$ and by considering the distribution of the DOF in the AT region (see further clarifications below), while $p\left(\boldsymbol{x}_{2}, N_{2} \mid N_{1}\right)$ is the distribution in the hybrid region, conditional on the number $N_{1}$ of particles in the atomistic region. We now comment on the underlying assumptions involved in the calculation of the conditional probability density.

(1) If we use the weighting function (1), then the AT region is interacting with the $\Delta$ region as if the $\Delta$ region were part of the AT region because all hybrid molecules that interact with the AT molecules have unit weight.

(2) We assume that $p\left(\boldsymbol{x}_{1} \mid N_{1} ; \boldsymbol{x}_{2}, N_{2}\right)$ can be approximated by

$$
p\left(\boldsymbol{x}_{1} \mid N_{1} ; \boldsymbol{x}_{2}, N_{2}\right) \propto e^{-\beta \mathcal{H}_{N_{1}}^{\mathrm{AT}}\left(\boldsymbol{x}_{1} ; \boldsymbol{x}_{2}, N_{2}\right)},
$$

where the Hamiltonian governing the physics of the molecules in the atomistic region is defined as

$$
\begin{aligned}
\mathcal{H}_{N_{1}}^{\mathrm{AT}}\left(\boldsymbol{x}_{1} ; \boldsymbol{x}_{2}, N_{2}\right) & \\
= & \sum_{j=1}^{N_{1}} \frac{1}{2} m_{i} \boldsymbol{v}_{i}^{2}+\sum_{i, j=1}^{N_{1}} \frac{1}{2} U^{\mathrm{AT}}\left(\boldsymbol{r}_{i}-\boldsymbol{r}_{j}\right) \\
& +\sum_{i=1}^{N_{1}} \sum_{j=N_{1}+1}^{N_{2}} U^{\mathrm{AT}}\left(\boldsymbol{r}_{i}-\boldsymbol{r}_{j}\right),
\end{aligned}
$$

which is exactly the same as for the equivalent subregion of the full atomistic system of reference. (The approximation is essentially based on the assumption that the AT and $\Delta$ regions are only shortrange correlated; the assumption is discussed in the following point.)

(3) We further assume that all interactions in the system have a finite range with a given cutoff radius; the electrostatic interaction is treated by the reaction-field method. Specifically, we suppose that the AT region does not interact in a direct way with the CG region. For the case of liquid water at ambient conditions, it is reasonable to assume that the system is only short-range correlated, i.e., that the AT region is only correlated with the $\Delta$ region. (We will show numerically the validity of the hypothesis of short-range correlation.) We suppose that all interactions satisfy the superstability condition of Ref. [15].

(4) The hypothesis of fixed molecules in the $\Delta$ region must not be intended in dynamical terms, which means that, during the dynamical evolution of the system, one should not suppose the molecules in the $\Delta$ region to be frozen in their positions while molecules in other regions are moving. Instead, this hypothesis must be intended in the sense of statistical analysis; within a statistical framework, it is intended that, for a given configuration of molecules in the $\Delta$ region, the AT region explores a large (statistical) number of configurations. In such a case, the practical statistical analysis consists of taking a given configuration in the $\Delta$ region. Then, one must consider the whole trajectory of a simulation and sort out all the configurations in the AT region that correspond to the given configuration in the $\Delta$ region. If one repeats the process for a large number of fixed configurations of the $\Delta$ region, then the data of the trajectory sorted according to this criterion would lead to a statistics for the AT region equivalent to that obtained by a sampling performed according to the given Hamiltonian of the AT region [Eq. (12)].

In accordance with the third assumption, we ignore correlations between the molecules of the $\Delta$ and CG regions; in fact, we even consider the DOF in the $\Delta$ region to be fixed while sampling configurations in the AT and CG regions. The question, then, is whether the probability $p\left(\boldsymbol{x}_{2}, N_{2} \mid N_{1}\right)$ in the $\Delta$ region is the same as that of the equivalent region in a fully atomistic reference system. In general, the probability distribution is not the same, so we state the necessary conditions to enforce such an equivalence to the lowest order in the configurational probability of the system; the necessary conditions are [4]

$$
\begin{aligned}
& \rho_{\Delta}(\boldsymbol{r})=\rho_{\mathrm{AT}}(\boldsymbol{r}), \\
& g_{\Delta}(r)=g_{\mathrm{AT}}(r) .
\end{aligned}
$$

The first-order marginal distribution is the particle (or molecular) density $\rho_{\Delta}(\boldsymbol{r})$, while the second-order marginal distribution is the RDF $g_{\Delta}(r)$. The necessary conditions of the correct distribution $p\left(\boldsymbol{x}_{1} \mid N_{1}\right)$ are that these two distributions should be the same as those of the fully atomistic reference system. These conditions are the minimal necessary ones that involve the basic DOF, that 
is, the molecular center-of-mass coordinates; however, one may require a more specific accuracy by imposing that any atom-atom $g(r)$ is also matched to the corresponding function of the atomistic reference system. In general, the conditions of Eq. (13) and (14) would assure that at least at the first- and second-order $p\left(\boldsymbol{x}_{2}, N_{2} \mid N_{1}\right)$ in the AdResS are the same as those of a full atomistic simulation. One needs to go at least at the second order, so that, at the interface between the atomistic and transition regions, the radial distribution functions of the atomistic part are not affected by artifacts due to the deviation of the RDFs of the $\Delta$ region from the correct atomistic reference. We have shown numerically how the RDF correction can be numerically implemented [4]. Higher accuracy can then be systematically reached by enforcing the equivalence of higher orders of the distribution in the transition region. Next, we must show that $p\left(N_{1}\right)$ is the same in AdResS and in the reference full atomistic simulation. The basic outline of arguments will be given in a following section, while the (long) explicit calculations are reported in Appendix A; however, before proceeding further, we must first treat a key ingredient of this equivalence, that is, the thermodynamic force. This force, in fact, together with the thermostat, is the crucial tool for assuring the thermodynamic and statistical equilibrium of the AdResS system when compared to the reference full atomistic simulation. In the next section, we will show how this force, derived on intuitive ground to enforce the equality of some basic thermodynamic relations, as shown in Eq. (4), is, together with the action of the thermostat, the key ingredient for the balance of chemical potential between the various regions at the desired density of equilibrium. The balance of chemical potential is implicitly a strong argument in favor of the idea of AdResS as a grand-canonical-like scheme (i.e., molecules are exchanged between the AT and CG regions in conditions of equilibrium).

\section{Thermodynamic force: From empirical intuition to strict formalization within the grand-canonical framework}

Intuitive thermodynamic considerations lead to formula (4). In this section, we show that, despite the fact that the argument used in Eq. 4 from Refs. [3,4] has a strong empirical component, one can formally justify this force as a tool to balance the chemical potential of the various resolutions and, as a consequence, a key aspect in interpreting AdResS as an effective grand-canonical setup. Here, two assumptions are made.

(1) $N_{2} \ll N_{1} \ll N_{3}$. The second inequality corresponds to the thermodynamic limit of the grandcanonical ensemble. The first one actually assumes that the $\Delta$ region is infinitely thin, so that it can be viewed as an infinitesimal membrane that allows a free exchange of molecules from the AT region to the $\mathrm{CG}$ region and vice versa.
(2) We have

$$
\mathcal{H}\left(\boldsymbol{x}_{1}, N_{1} ; \boldsymbol{x}_{3}, N_{3}\right)=\mathcal{H}_{N_{1}}^{\mathrm{AT}}\left(\boldsymbol{x}_{1}\right)+\mathcal{H}_{N_{3}}^{\mathrm{CG}}\left(\boldsymbol{x}_{3}\right)
$$

The equation above is reasonable if the system is short-range correlated.

The equilibrium in the AT and CG regions is assured by the membrane $\Delta$ via the action of thermodynamic force and of the thermostat. Conceptually, we assume that there is an infinitely thin "filter" (see Fig. 2) located at the interface between the AT and CG regions. When a molecule enters into the AT region or leaves it, the filter does some work per molecule, $\omega_{0}$, on the atomistic system in order to assure the thermodynamic equilibrium. Therefore, we add an empirical term in the Hamiltonian of the system $N_{1} \omega_{0}$, related to the work done by the filter to obtain configurations of the atomistic region with $N_{1}$ molecules. Having fixed the number of molecules, that is, ideally considering caseby-case situations at fixed $N_{1}$ and $N_{3}$, and by following the arguments of the last section, both the AT and the CG regions are subject to the Boltzmann distribution. Thus, the fixed-number partition function $\left(N_{1}\right.$ in the AT region and $N_{3}$ in the CG region) of the system reads

$$
\begin{aligned}
q(N, V, T)= & \frac{1}{N !} \int d \boldsymbol{x} e^{-\beta\left[\mathcal{H}\left(x_{1}, N_{1} ; x_{3}, N_{3}\right)+N_{1} \omega_{0}\right]} \\
= & \frac{1}{N !} \int d x_{1} d x_{3} e^{-\beta\left[\mathcal{H}_{N_{1}}^{\mathrm{AT}}\left(\boldsymbol{x}_{1}\right)+\mathcal{H}_{N_{3}}^{\left.\mathrm{CG}\left(x_{3}\right)+N_{1} \omega_{0}\right]}\right.} \\
= & \frac{N_{1} ! N_{3} !}{N !} e^{-\beta N_{1} \omega_{0}} \frac{1}{N_{1} !} \int d \boldsymbol{x}_{1} e^{-\beta \mathcal{H}_{N_{1}}^{\mathrm{AT}}\left(x_{1}\right)} \\
& \times \frac{1}{N_{3} !} \int d \boldsymbol{x}_{3} e^{-\beta \mathcal{H}_{N_{3}}^{\mathrm{CG}\left(x_{3}\right)}} \\
= & \frac{N_{1} ! N_{3} !}{N !} e^{-\beta N_{1} \omega_{0}} Q_{\mathrm{AT}}\left(N_{1}, V_{1}, T\right) Q_{\mathrm{CG}}\left(N_{3}, V_{3}, T\right) .
\end{aligned}
$$

Considering the permutations of particles, the number of possibilities of $N_{1}$ molecules in the atomistic region and $N_{3}$ molecules in the coarse-grained region is $\frac{N !}{N_{1} ! N_{3} !}$. Therefore, the partition function of the whole system reads

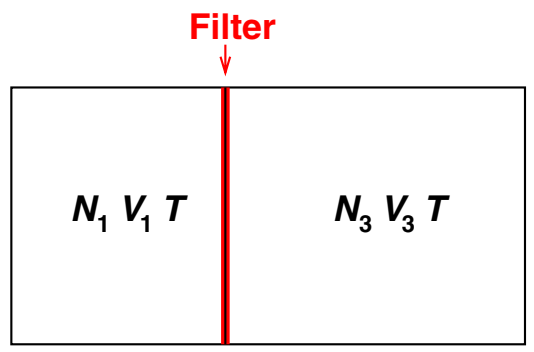

FIG. 2. Schematic plot of the AdResS system in thermodynamic equilibrium. The thickness of the filter corresponds to $d_{\Delta}$. 


$$
\begin{aligned}
Q(N, V, T)= & \sum_{N_{1}=1}^{N} \frac{N !}{N_{1} ! N_{3} !} \frac{N_{1} ! N_{3} !}{N !} e^{-\beta N_{1} \omega_{0}} Q_{\mathrm{AT}}\left(N_{1}, V_{1}, T\right) \\
& \times Q_{\mathrm{CG}}\left(N_{3}, V_{3}, T\right) \\
= & \sum_{N_{1}=1}^{N} e^{-\beta N_{1} \omega_{0}} Q_{\mathrm{AT}}\left(N_{1}, V_{1}, T\right) Q_{\mathrm{CG}}\left(N_{3}, V_{3}, T\right),
\end{aligned}
$$

with natural relations

$$
\begin{aligned}
& N=N_{1}+N_{3}, \\
& V=V_{1}+V_{3} .
\end{aligned}
$$

For convenience, we denote

$$
\tilde{q}_{N_{1}}=e^{-\beta N_{1} \omega_{0}} Q_{\mathrm{AT}}\left(N_{1}, V_{1}, T\right) Q_{\mathrm{CG}}\left(N-N_{1}, V_{3}, T\right) .
$$

Further, let $\bar{N}_{1}$ be the value at which $\tilde{q}_{N_{1}}$ reaches its unique maximum, namely, $\tilde{q}_{\bar{N}_{1}}=\max \tilde{q}_{N_{1}}$. (The existence of a maximum follows from the superstability of the interactions that guarantees that particles do not cluster as $N \rightarrow \infty$; we further assume that it is unique.) Since $\tilde{q}_{N_{1}}$ is positive definite, a basic observation is that

$$
\tilde{q}_{\bar{N}_{1}} \leq \sum_{N_{1}=1}^{N} \tilde{q}_{N_{1}} \leq N \tilde{q}_{\bar{N}_{1}}
$$

Because of the monotonicity of the logarithm, we have

$$
\ln \tilde{q}_{\bar{N}_{1}} \leq \ln \sum_{N_{1}=1}^{N} \tilde{q}_{N_{1}} \leq \ln \left(N \tilde{q}_{\bar{N}_{1}}\right)=\ln N+\ln \tilde{q}_{\bar{N}_{1}} .
$$

If $\ln N \ll \ln \tilde{q}_{\bar{N}_{1}}$, Laplace's method yields (see, e.g., Ref. [16], Sec. 4.3)

$$
\ln \sum_{N_{1}=1}^{N} \tilde{q}_{N_{1}} \approx \ln \tilde{q}_{\bar{N}_{1}} .
$$

(The validity of the antecedent will be discussed later.) Hence,

$$
\begin{aligned}
\ln Q(N, V, T) \approx & -\beta \bar{N}_{1} \omega_{0}+\ln Q_{\mathrm{AT}}\left(\bar{N}_{1}, V_{1}, T\right) \\
& +\ln Q_{\mathrm{CG}}\left(N-\bar{N}_{1}, V_{3}, T\right)
\end{aligned}
$$

or, equivalently,

$$
A(N, V, T) \approx \bar{N}_{1} \omega_{0}+A_{\mathrm{AT}}\left(\bar{N}_{1}, V_{1}, T\right)+A_{\mathrm{CG}}\left(N-\bar{N}_{1}, V_{3}, T\right),
$$

where $A$ denotes the Helmholtz free energy. At this point, the crucial question is if the condition $\ln N \ll \ln \tilde{q}_{\bar{N}_{1}}$ holds or, equivalently,

$$
\begin{aligned}
\ln N \ll & -\beta \bar{N}_{1} \omega_{0}+\ln Q_{\mathrm{AT}}\left(\bar{N}_{1}, V_{1}, T\right) \\
& +\ln Q_{\mathrm{CG}}\left(N-\bar{N}_{1}, V_{3}, T\right) .
\end{aligned}
$$

Generally, the condition above is true: $\ln Q_{\mathrm{CG}}(N-$ $\left.\bar{N}_{1}, V_{3}, T\right)$ is proportional to the free energy $A_{\mathrm{CG}}(N-$ $\left.\bar{N}_{1}, V_{3}, T\right)$, which is an extensive thermodynamic variable, so $A_{\mathrm{CG}}\left(N-\bar{N}_{1}, V_{3}, T\right)$ is proportional to $N-\bar{N}_{1}$. Because of the thermodynamic limit $N \gg \bar{N}_{1}, A_{\mathrm{CG}}\left(N-\bar{N}_{1}, V_{3}, T\right)$ is proportional to $N$, which is much larger than $\ln N$ for $N \gg 1$; the arguments just given validate condition (26). We will see later [from Eq. (A1)] that the maximum $\bar{N}_{1}$ corresponds to the maximum value of probability $p\left(N_{1}\right)$; $\bar{N}_{1}$ is also the average molecule number in the AT region that is of statistical importance under the thermodynamic limit. As the right-hand side of Eq. (25) attains its maximum at $\bar{N}_{1}$ when the other thermodynamic variables are kept fixed, differentiating it with respect to $N_{1}$ entails

$$
\omega_{0}=\mu_{\mathrm{CG}}\left(N-\bar{N}_{1}, V_{3}, T\right)-\mu_{\mathrm{AT}}\left(\bar{N}_{1}, V_{1}, T\right) .
$$

The equation above means that the difference in the chemical potential between the AT and CG regions is taken care of by the work of the filter. Now, if the filter ensures an equilibrium that is the same as the full atomistic reference system, then

$$
\omega_{0}=\mu_{\mathrm{CG}}\left(\rho_{0} V_{3}, V_{3}, T\right)-\mu_{\mathrm{AT}}\left(\rho_{0} V_{1}, V_{1}, T\right) .
$$

$\rho_{0}$ is the number density at which the atomistic and coarsegrained resolutions should match; namely, $\bar{N}_{1}=\rho_{0} V_{1}$ and $N-\bar{N}_{1}=\rho_{0}\left(V-V_{1}\right)$ should apply. Equation (28) is a necessary condition for the work required to the filter in order to have the correct equilibrium of the AT and CG regions.

As anticipated in the previous sections, the work of the filter has two components: one that corresponds to the work of the thermodynamic force and another that corresponds to the work of the thermostat, i.e., $\omega_{0}=\omega_{\text {th }}+\omega_{Q}$. We indicate with $\omega_{\text {th }}$ the work of the thermodynamic force and with $\omega_{Q}$ that of the thermostat in the transition region. The existence of $\omega_{Q}$ has been numerically proven in Ref. [17] and can be decomposed into two parts: $\omega_{Q}=\omega_{\text {DOF }}+$ $\omega_{Q}^{\text {extra }} \cdot \omega_{\text {DOF }}$ is related to the thermalization of the degrees of freedom (rotational and/or vibrational) that are reintroduced or removed, and, according to the equipartition theorem, one has $\frac{1}{2} k_{B} T$ per degree of freedom, while $\omega_{Q}^{\text {extra }}$ is due to the absence of the energy conservation that is related to the different intermolecular interactions in the different regions of the system, as is shown in Ref. [13]. In Appendix D, we will show how this term can be numerically calculated within a standard AdResS, thus allowing the explicit calculation of the chemical potential of a system.

We remind the reader that the argument in this section is essentially based on a large deviation principle (namely, Laplace's method) for the number of particles in the AT region that entails that $\bar{N}_{1}$ rather than $N_{1}$ can be considered as a representative of the configuration realizations in the AT region. The assumption above implies that the particle fluctuations, $\Delta N_{1}$, are negligible compared to $\bar{N}_{1}$ in the AT region. This hypothesis is valid if the AT region is large enough so that $\bar{N}_{1}$ is large. This point seems to be true in all numerical experiments that have been done so far (see, e.g., Ref. [18]). In this way, we have shown the formal derivation 
of the thermodynamic force and the thermostat as tools to balance the chemical potential between the two regions. For a grand-canonical-like setup, the balance of chemical potential between open regions is a necessary condition to have the exchange of molecules in thermodynamic equilibrium, and, as a consequence, we have formally justified why Eq. (4) is a necessary condition for AdResS to be considered an effective grand-canonical setup.

\section{The number probability $p\left(N_{1}\right)$}

In this section, we provide the basic arguments by which one can define at which level of approximation $p\left(N_{1}\right)$ in AdResS is the same as in a full atomistic simulation. This equality is an important point in order to justify the reliability of AdResS as a grand-canonical setup in terms of probability distribution. In fact, if $p\left(N_{1}\right)$ in AdResS is very different from the corresponding one of a full atomistic simulation, then clearly the AdResS method cannot be considered valid since the artifacts due to the different $p\left(N_{1}\right)$ would give an unrealistic description of the system. Essentially, there are two arguments of this equivalence. The first-order accuracy of $p\left(N_{1}\right)$ requires the balance of the chemical potential:

$$
\mu_{\mathrm{CG}}=\mu_{\mathrm{AT}}+\omega_{0} .
$$

The relation above, in the previous section, has been shown to hold because of the action of the thermodynamic force and the thermostat in the thermodynamic limit. Whether or not the size of standard and feasible molecular simulations can be considered, effectively, in the thermodynamic limit will be checked later on with numerical tests; however, we can anticipate that the answer is positive for systems whose size and time of simulation are nowadays routinely done. The second order of accuracy requires equality for the compressibility:

$$
\kappa_{\mathrm{CG}}=\kappa_{\mathrm{AT}}
$$
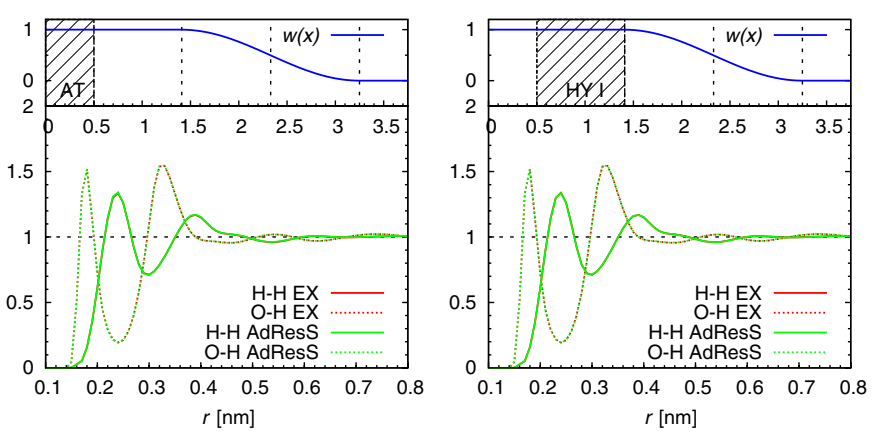

Equation (30) implies that the COM-COM RDFs of the atomistic and coarse-grained regions are matched [19]. Essentially, Eqs. (29) and (30) are the physical requirements to show that, up to the second order, $p\left(N_{1}\right)$ in AdResS is the same as in the equivalent subregion of the full atomistic system of reference. Because of the lengthy arguments, specific details are reported in Appendix A.

\section{A NUMERICAL TEST: LIQUID WATER}

The arguments we have used so far are not sufficient to infer that the probability $p\left(\boldsymbol{x}_{1}, N_{1}\right)$, as presented in the theoretical analysis, corresponds to that of a grandcanonical distribution [Eq. (5)]. One must be very careful about any statement on $p\left(\boldsymbol{x}_{1}, N_{1}\right)$ because of the following three reasons.

(1) There is no hope whatsoever that the assumed Boltzmann form of the distribution $p\left(\boldsymbol{x}_{1} \mid N_{1} ; \boldsymbol{x}_{2}, N_{2}\right)$ can be numerically verified for any realistic test system.

(2) Conditions (13) and (14) are only necessary and are, in general, not sufficient to guarantee the correct distribution $p\left(\boldsymbol{x}_{2}, N_{2} \mid N_{1}\right)$ in the hybrid region $\Delta$.

(3) Formally, the particle number density $p\left(N_{1}\right)$ is only a second-order approximation of the correct density $p_{\mathrm{AT}}\left(N_{1}\right)$.

However, the number distribution $p\left(N_{1}\right)$ has already been proven to be numerically correct in Refs. [3,4] for the relevant case of liquid water. In this section, we want to test, with a numerical simulation, to what extent other quantities, e.g., the configurational distribution $p\left(\boldsymbol{x}_{1}\right)=$ $\sum_{N_{1}} p\left(\boldsymbol{x}_{1}, N_{1}\right)$, are correct. Obviously, it is a prohibitive task for any complex molecular system to determine the high-dimensional configurational distribution; for this reason, instead, we study its marginal distributions up to the third order. Here, we report the $\mathrm{H}-\mathrm{O}$ and $\mathrm{H}-\mathrm{H}$ RDFs (see Fig. 3) and the three-body COM correlation function
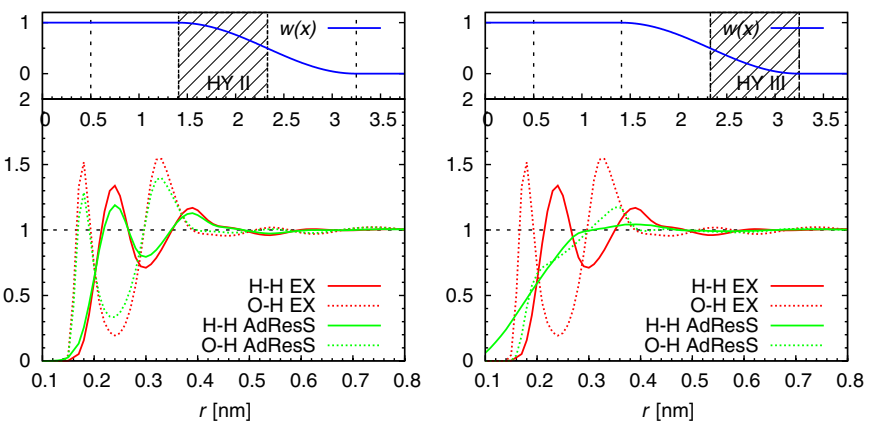

FIG. 3. Local H-H and O-H $g(r)$ 's. The red line is the curve that corresponds to the reference explicit (all-atom) simulation (EX). The curve obtained by employing the AdResS method is represented in green. The hybrid region is equally divided into three parts: HY I, HY II, and HY III, the widths of which are roughly equal to the cutoff radius, i.e., $9 \mathrm{~nm}$. The top part of each panel shows the region where the $g(r)$ is calculated and the value of the weighting function $w(x)$ in each region. From left to right, the panels correspond to the AT region, the HY I subregion of $\Delta$, the HY II subregion of $\Delta$, and the HY III subregion of $\Delta$, respectively. It can be seen that, beyond $0.5 \mathrm{~nm}$, the functions go to 1; that is, particles beyond this distance are uncorrelated. The result is fully consistent with our hypothesis of Eq. (11). 

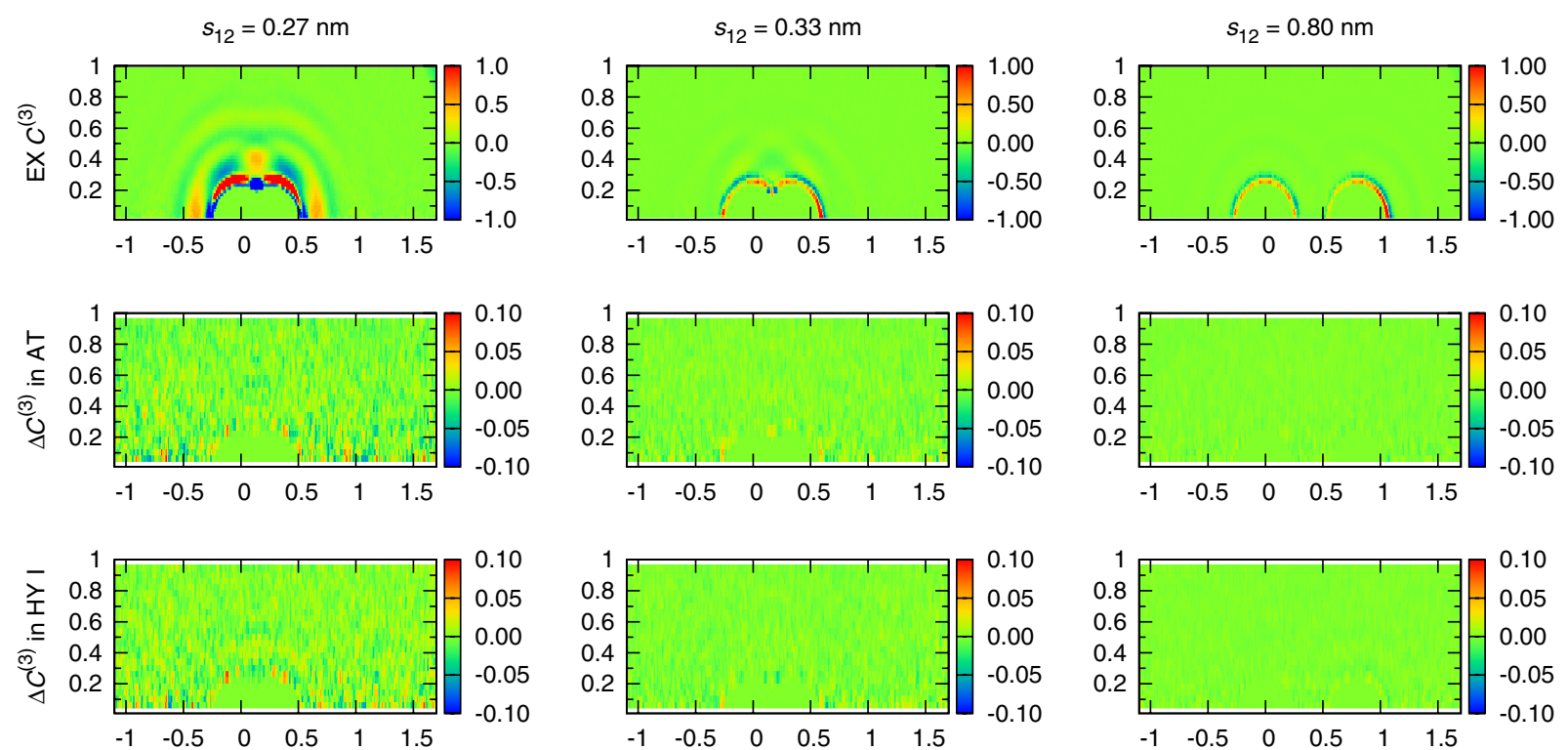

FIG. 4. The three-body correlation function $C^{(3)}$. The first row corresponds to the EX. The second and third rows show the difference between the function calculated in AdResS in the AT and HY I regions, respectively, and the reference function of the full atomistic case. Different columns give different distances between the first two molecules: From left to right, $s_{12}=0.27,0.33$, and $0.80 \mathrm{~nm}$. The horizontal axis corresponds to the variable $h_{1}$, while the vertical axis is the variable $h_{2}$. (See Appendix C for the definition of these variables.) The magnitude of the correlation and the differences are indicated by different colors.

$C^{(3)}\left(s_{1}, s_{2}, s_{2}\right)$ (see Fig. 4). For simulation protocols and the definition of $C^{(3)}$, see Appendixes B and C. The first-order marginal distribution, that is, the molecular density profile, and the second-order marginal distribution, that is, the COM RDF, are not presented here; we refer readers to Ref. [4]. Here, we focus on the more delicate RDFs that involve the atomistic accuracy and on the third-order COM three-body correlation function $\left(C^{(3)}\right)$. From Fig. 3, one can see that the AdResS H-O and H-H RDFs are identical to those of the full atomistic reference system in the AT region. Interestingly, despite the fact that the corrective force of the RDF is applied only to the COM RDF, also in the region HY I (that is close to the atomistic region), the $\mathrm{H}-\mathrm{O}$ and H-H RDFs are the same as in the full atomistic case. The equality of RDFs implies that the AT region is embedded in an environment where not only the COMCOM structural properties but also the finer structural properties are the same as for the atomistic resolution. In the HY II and III regions, the AdResS results obviously deviate from those of the full atomistic reference system because the atomistic nature is decreasing as a molecule travels from HY I to HY III, so it must be expected that the orientational order is also fading away. In HY III, the H-O and H-H RDFs are almost structureless. Furthermore, we test $C^{(3)}$ in Fig. 4. (For the definition of the three-body correlation function $C^{(3)}$, see Appendix C.) Interestingly, the three-body correlation is correctly reproduced by the AdResS in the AT and HY I regions. Such a result is a further strong argument in favor of the fact that, in AdResS, the AT region is embedded in an environment that, at least up to the three-body COM correlation, is the same as the full atomistic reference system. In the HY II and HY III regions, the three-body correlation deviates from the full atomistic reference; in fact, since the various $g(r)$ already deviates, one cannot expect a higher-order correlation function to match (if not by chance) the full atomistic function of reference. Another important piece of information in Figs. 3 and 4 is that the hypothesis of the short-range influence of a region over another employed in Eq. (11) is numerically justified. Thus, we can state that, for the case of liquid water at ambient conditions, the AT region is only short-range correlated with the rest of the system.

\section{WCA POTENTIAL INSTEAD OF ATOMISTIC-BASED COARSE-GRAINED POTENTIAL: COUPLING THE ATOMISTIC REGION TO A GENERIC SOURCE OF ENERGY AND PARTICLES}

The numerical test presented in the previous section shows how an atomistic model and its corresponding coarse-grained model, which resemble the basic structural properties of the original full atomistic system, can be interfaced in an adaptive way and exchange molecules in a grand-canonical fashion. However, one may be tempted to think that the request of having a coarse-grained model that resembles as much as possible the structural and thermodynamic properties of a full atomistic model would be a necessary condition to have a proper exchange of particles between the different regions. If it was so, the idea of the adaptive scheme as a grand-canonical tool would not be very general; our theoretical results instead 
suggest that it must be sufficient to impose some given conditions in the transition region to have a proper exchange of energy and particles between the small atomistic region and the large coarse-grained reservoir. If the theoretical considerations are correct, one should be able to show numerically that the proper exchange of energy and molecules is independent from the molecular model used in the coarse-grained region; that is, the filter of the transition region makes the atomistic region a grand-canonical ensemble. For this reason, we have carried out a further numerical test where the molecules in the coarse-grained region interact with a generic WCA potential, which assures that only the density, but not the radial distribution function, of a liquid governed by this potential is the same as that of the liquid water. (For the simulation setup, see Appendix B.)

The left plot of Fig. 5 shows that the density profile of the WCA AdResS perfectly matches the all-atom reference system; satisfying agreement is also found in the AT and HY I regions of the right plot of Fig. 5, which shows the molecular number fluctuation over the whole system. Figure 6 shows the COM-COM, H-O, and H-H RDFs in the AT, HY I, HY II, and HY III regions. All the RDFs are compared with the full atomistic reference system (EX). Finally, Fig. 7 compares the three-body correlation $C^{(3)}$ with the atomistic reference system and plots the difference. It must be noticed that, in this case, we intentionally want to investigate a "worst-case scenario," and thus we did not apply the RDF corrective force in the transition region. In this way, we can understand whether or not the corrective action of the thermodynamic force only is sufficient from the numerical point of view to reproduce the grand-canonical-like environment for the AT region. According to our theoretical framework, in the absence of the RDF corrective force, only the first order of the distribution (i.e., the density profile) is corrected by the thermodynamic force in the $\Delta$ region. However, numerically, Fig. 6 shows a second-order accuracy (correct RDFs) in the HY I region, as well. Instead, the three-body correlation in HY I deviates from the full atomistic reference; the deviation is the price we pay for this worst-casescenario setup. Nevertheless, the accuracy reached in the transition region is sufficient in order to have, at least up to the second order, the AT region of the WCA AdResS
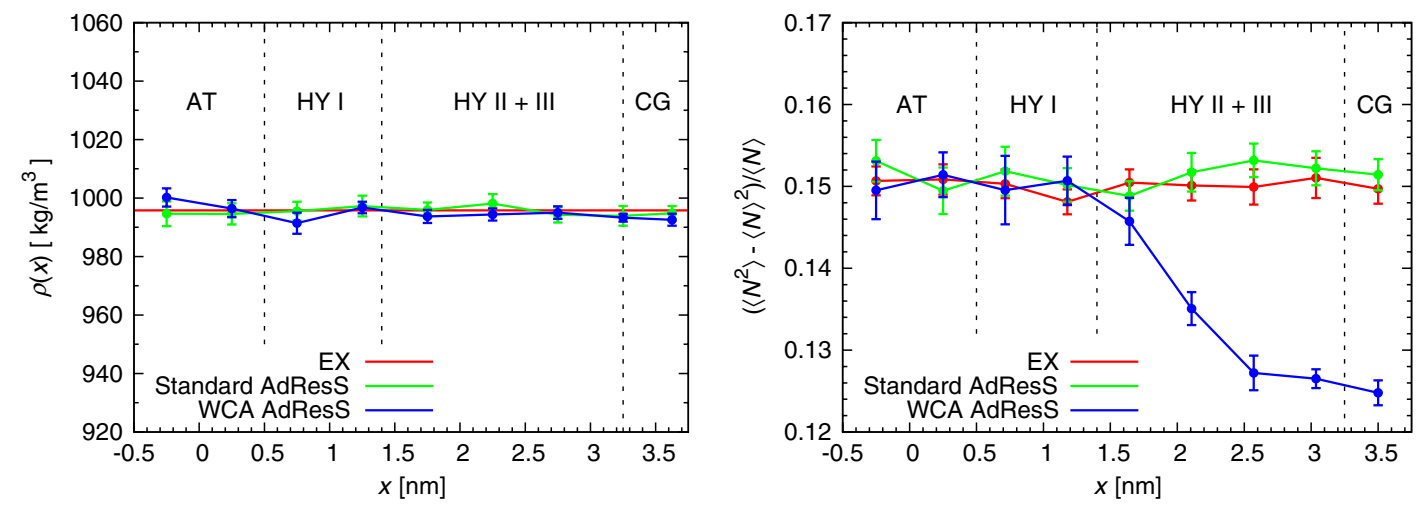

FIG. 5. Density and fluctuation profiles of the case of the WCA potential in AdResS. The results are compared with the EX and the standard AdResS. The error bars indicate the statistical error up to a confidence level of $95 \%$.
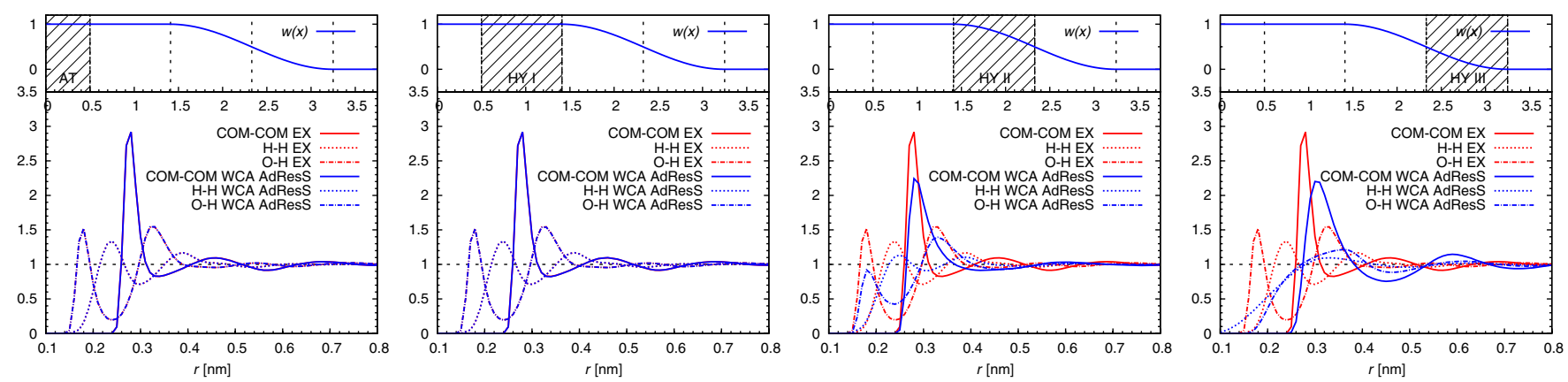

FIG. 6. The COM-COM, H-O, and H-H RDFs. The red line is the curve that corresponds to the EX. The curve obtained by employing the WCA AdResS method is represented in blue. The hybrid region is equally divided into three parts: HY I, HY II, and HY III, the widths of which are roughly equal to the cutoff radius, i.e., $9 \mathrm{~nm}$. The top part of each panel shows the region where the $g(r)$ is calculated and the value of the weighting function $w(x)$ in each region. From left to right, the panels correspond to the AT region, the HY I subregion of $\Delta$, the HY II subregion of $\Delta$, and the HY III subregion of $\Delta$, respectively. 

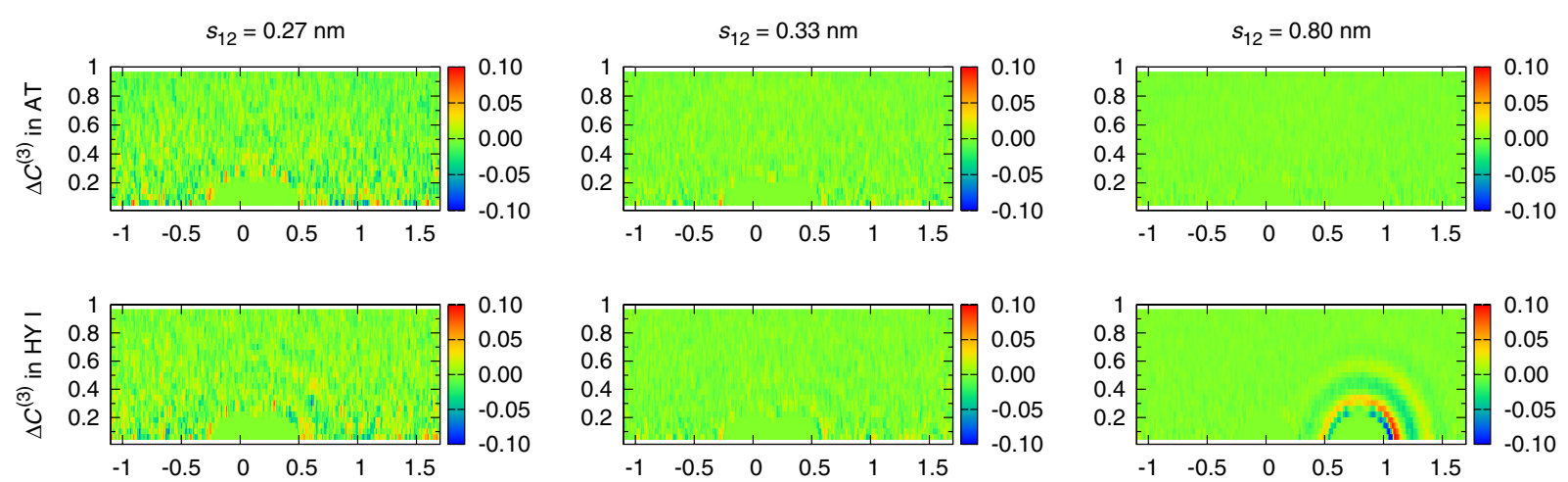

FIG. 7. The three-body correlation of the WCA AdResS in the AT and HY I regions. The difference with respect to the full atomistic reference (see the first line of Fig. 4) is plotted in this figure. From left to right, the distances between the first two molecules are $s_{12}=0.27,0.33$, and $0.80 \mathrm{~nm}$, respectively.

equivalent to the corresponding subregion in the full atomistic reference. Furthermore, numerical results (Fig. 7) show that, in the AT region, even the three-body correlation $C^{(3)}$ is nonetheless very accurate. The accuracy of the AdResS results means that, from the numerical point of view, only the work of the thermodynamic force and that of the thermostat, by allowing an exchange of particles under conditions of equilibrium of the chemical potential, is sufficient to assure the correct equilibrium of the atomistic region. The possible application of the corrective force on the RDFs in $\Delta$ in the case of liquid water is, in this case, numerically not required, but, in case of necessity, it would nevertheless represent a systematic tool for improving accuracy. Summarizing, the results reported in Figs. 5-7 show that, in the atomistic region, the accuracy at the third order and particle number fluctuations, for which the filter in the transition region is designed, are well preserved; that is, the atomistic region exchanges particles and energy with the reservoir in a proper grand-canonical fashion (up to the level of approximation decided a priori). As the molecules in the coarse-grained region are a generic liquid model, we have proven numerically that the adaptive technique employed here can indeed be considered as a tool for general grand-canonical molecular-dynamics simulations.

\section{COMPUTATIONAL EFFICIENCY OF AdResS AS A GRAND-CANONICAL SETUP}

In order to show the computational efficiency of the AdResS method as a grand-canonical setup, we have carried out a numerical experiment to compare the performance of our method in inserting and removing molecules from the atomistic region and that of a highly popular approach used in molecular dynamics, the IPM [20]. The IPM is very efficiently implemented into the GROMACS code [21], and thus it is a natural choice as a grand-canonical-like setup for a very large portion of molecular-dynamics simulators. The experiment consists of the following: We have considered a large full atomistic system and applied the highly optimized IPM to insert molecules. From this study, we extract the efficiency of the IPM in terms of the required computational resources. Next, we consider an adaptive system whose number of molecules, in the atomistic-plus-hybrid region, is at least the same as the full atomistic system considered. (Actually, for the results presented here, the size considered is even larger than that of the EX simulation.) To the atomisticplus-hybrid region, we add a rather large reservoir of coarse-grained particles. (Tests were also carried out with smaller reservoirs, but the results were essentially the same as for the large reservoir.) Also, in this case, the efficiency is measured in terms of computational resources required. The results show that the IPM procedure itself requires a rather high computational cost, to which long trajectories must be added, so that the insertion of one molecule per time converges, and data can be then collected for real statistics. Instead, the adaptive approach performs, in less time and at a much lower computational cost, on-the-fly dynamical multiple insertions, keeping the instantaneous equilibrium intact, which implies the additional advantage that data can be collected on the fly for real statistics at any time. Specifically, we use an equilibrium trajectory of length $8 \mathrm{~ns}$, where the coordinates of water molecules are recorded every 0.2 ps. Using the IPM approach, in each frame, $10^{5}$ test particles are inserted at random positions of the system. For each insertion, $10^{5}$ randomly chosen orientations are tried. The convergence is very slow-even at $8 \mathrm{~ns}$, the insertion process is not satisfactorily converged (see Fig. 8). Lack of convergence of the insertion process means that, up to this point, data cannot be collected for analysis of physical properties. Compared to the IPM, AdResS leads to a very efficient insertion of particles: In each 1-ps time interval, (on average) 23 water molecules enter into the atomistic region. Some of them come back immediately because of an unfavorable entering configuration. To investigate the proper insertion, we consider the time interval of $1 \mathrm{~ns}$ and find that 832 out of 13824 molecules are first in either the HY or CG regions and 

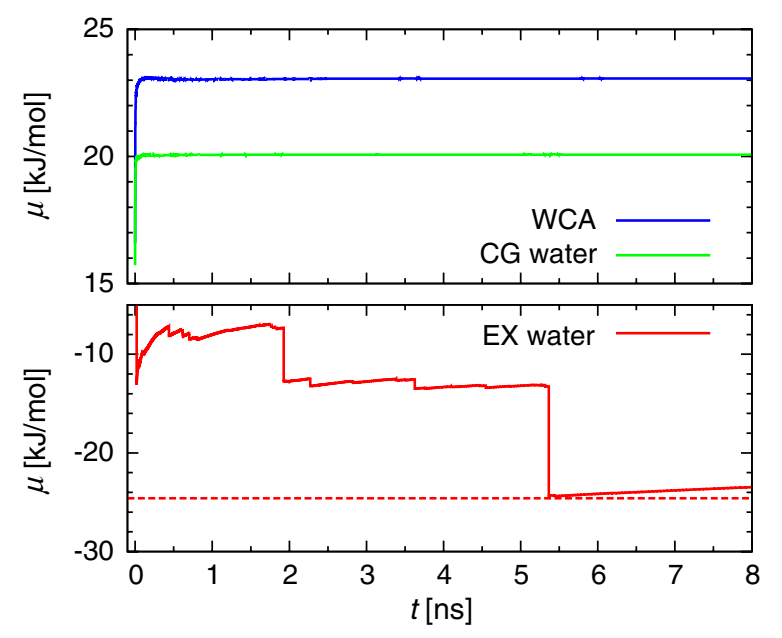

FIG. 8. The chemical potential calculated by the IPM. The top panel shows the case of CG water and the WCA liquid, while the bottom panel shows the case of an EX simulation. The horizontal dashed line in the bottom panel is used to guide the eyes and represents the ideal target. The plot shows the result that, for EX water, the IPM procedure does not converge within $8 \mathrm{~ns}$; in fact, the convergence of the chemical potential means that the insertion of the molecule is properly done, and a subsequent part of the trajectory (with the associated IPM) can be used for a "statistical" average in a grand-canonical framework.

then that they travel to the AT region and stay there for longer than $60 \mathrm{ps}$, which indicates a root-mean-square displacement of roughly $1 \mathrm{~nm}$ in the AT region. Moreover, while for the EX simulation one must avoid size effects by choosing reasonably large systems, in AdResS, the AT region can actually be chosen to be much smaller than the one used here, thus enhancing the amount of efficiency. Finally, even if one can afford a rather large atomistic system, and thus a faster convergence, the cost of the insertion procedure will increase enormously because it would require a larger sampling due to the increased number of possibilities to allocate a particle in the liquid. These results, in terms of computational resources, are summarized in Table I. Moreover, in previous work, Praprotnik et al. [22] have shown that the coarse-grained region of AdResS can be easily coupled to the continuum; the results of Fig. 8 have the nontrivial advantage that the insertion of a sphere in a liquid of spheres by the IPM is, by far, much simpler than inserting an atomistically structured molecule in a liquid of atomistic molecules. Figure 8 shows the extremely fast convergence for inserting a particle in our "reservoir" of spherical particles compared to that of a full atomistic system. The results of Fig. 8 mean that, in the long-term perspective, the part of the algorithm of Praprotnik et al. could be merged with our current approach and thus allow for even smaller sizes of the reservoir region. However, this extension, although conceptually straightforward, goes beyond the scope of this paper. In general, there have been a large number of developments for the grand-canonical idea based on the "grow-in" and "shrinkout" moleculardynamics approaches (see, e.g., Lynch and Pettitt [10] and Eslami and Müller-Plathe [11]). However, all of them share the same basic principle and face the same problem of the IPM. This numerical experiment shows that, despite the need of a reservoir, our computation is by far more efficient than any other technique used so far. The comparison between the performance of AdResS and the IPM as a grand-canonical setup leads to the natural question of whether or not our method can be employed to calculate the excess chemical potential, which is the primary purpose for which the IPM is used. If this calculation of the excess chemical potential is possible, then AdResS may also represent a more efficient computational tool than the IPM for calculating such a quantity. In Appendix D, we provide a practical scheme for using AdResS as a tool to calculate the chemical potential and show that the results are in rather satisfying agreement with those obtained by the IPM.

\section{CONCLUSIONS}

We have shown a detailed theoretical analysis of the validity, limitations, and meaning of the AdResS method

TABLE I. Comparison of computational efficiency. The AdResS simulation contains 13824 molecules, while the EX water simulations contain 1728 molecules. In the AdResS simulation, the size of the AT + HY regions $\left(6.7 \times 3.7 \times 3.7 \mathrm{~nm}^{3}\right)$ is even larger than that of the EX water simulation box, so that we are actually in a worst-case-scenario situation. Moreover, simulations with smaller reservoirs give essentially the same results reported for this system. "System size" means the size of the box used in the simulation. "Trajectory length" means the equilibrium trajectory used for the particle insertion in the IPM approach. Along the trajectories, frames of configurations are recorded every $0.2 \mathrm{ps}$. "CPU time" is the wall clock time spent on the simulation. For the particle insertion simulation, the CPU time is counted in two parts: the time of generating the equilibrium trajectories (not expensive) and the time required by the IPM procedure (very expensive). Moreover, not only is the insertion procedure itself expensive, but even after $8 \mathrm{~ns}$ the insertion process has not actually converged. Simulations on longer time scales show that the full convergence is actually never reached, which suggests that the insertion procedure for a molecule as simple as water is not fully rigorous from the physical point of view.

\begin{tabular}{lccc}
\hline \hline & System size $\left(\mathrm{nm}^{3}\right)$ & Trajectory length $(\mathrm{ns})$ & CPU time (hours) \\
\hline AdResS & $29.9 \times 3.7 \times 3.7$ & 1 & 3.1 \\
EX water & $3.7 \times 3.7 \times 3.7$ & 8 & 4.5 (trajectory) +36.7 (IPM) \\
\hline \hline
\end{tabular}


within the framework of a grand-canonical ensemble. Using strict formal arguments, we have demonstrated the role of the thermodynamic force and of the thermostat in balancing the difference in chemical potential due to the different resolutions in space. The theoretical results, derived under the assumption of a thermodynamic limit, are then checked with several numerical tests. These latter numerical tests represent a worst-case scenario since the conditions of simulation are not ideal, as in the analytical procedure. Next, we have shown that, under the given hypothesis, $p\left(N_{1}\right)$, the probability distribution in the atomistic region of AdResS is equivalent to that in the same region in a full atomistic simulation up to the accuracy of the second order. We have further strengthened our hypothesis and conclusions by carrying out a numerical experiment for the case of liquid water at ambient conditions, where we could even go beyond the COM RDF. A further numerical experiment is made where the atomistic-based coarse-grained model is substituted by a generic liquid of spheres that interact via the WCA potential. The results show that the accuracy in the atomistic region is the same as that of AdResS, based on a coarsegrained model that is derived from the full atomistic reference system; these results strengthen the idea of AdResS as a tool for coupling the atomistic region to a generic source of energy and particles in a grand-canonical manner. Finally, we validate the efficiency of our method as a grandcanonical setup, comparing its computational performance to that of well-established and largely used alternative methods. In conclusion, these results provide a solid theoretical basis to explain the numerical reliability of the AdResS as an effective grand-canonical setup and to provide a basis for further formal and numerical development of the adaptive idea in terms of matching probability distributions.

\section{ACKNOWLEDGMENTS}

This work was partially supported by the Deutsche Forschungsgemeinschaft (DFG), with the Heisenberg Grant provided to L.D. S. (Grant No. DE 1140/5-1), and by ITN Nanopoly provided to H. W. and C. S. It was also supported in part by the National Science Foundation under Grant No. NSF PHY11-25915.

\section{APPENDIX A: THE NUMBER PROBABILITY $p\left(N_{1}\right)$}

In this section, we define at which level of approximation $p\left(N_{1}\right)$ in AdResS is the same as in a full atomistic simulation. Let us consider the marginal distribution $p\left(N_{1}\right)$,

$$
\begin{aligned}
p\left(N_{1}\right) & =\frac{N !}{N_{1} ! N_{3} !} \int d \boldsymbol{x}_{1} d \boldsymbol{x}_{3} \frac{1}{Q(N, V, T) N !} e^{-\beta\left[\mathcal{H}_{N_{1}}^{\mathrm{AT}}\left(\boldsymbol{x}_{1}\right)+\mathcal{H}_{N_{3}}^{\left.\mathrm{CG}\left(x_{3}\right)+N_{1} \omega_{0}\right]}\right.} \\
& =\frac{N !}{N_{1} ! N_{3} !} \frac{N_{1} ! N_{3} !}{Q(N, V, T) N !} e^{-\beta N_{1} \omega_{0}}\left[\frac{1}{N_{1} !} \int d \boldsymbol{x}_{1} e^{-\beta \mathcal{H}_{N_{1}}^{\mathrm{AT}}\left(\boldsymbol{x}_{1}\right)}\right]\left[\frac{1}{N_{3} !} \int d \boldsymbol{x}_{3} e^{-\beta \mathcal{H}_{N_{3}}^{\mathrm{CG}\left(\boldsymbol{x}_{3}\right)}}\right] \\
& =\frac{e^{-\beta N_{1} \omega_{0}} Q_{\mathrm{AT}}\left(N_{1}, V_{1}, T\right) Q_{\mathrm{CG}}\left(N-N_{1}, V_{3}, T\right)}{\sum_{n_{1}} e^{-\beta n_{1} \omega_{0}} Q_{\mathrm{AT}}\left(n_{1}, V_{1}, T\right) Q_{\mathrm{CG}}\left(N-n_{1}, V_{3}, T\right)}
\end{aligned}
$$

where $n_{1}$ is the summation variable that goes from 0 to $N$. In any case, when $n_{1}$ is not much smaller than $N$ (i.e., out of the range of the thermodynamic limit), the statistic is not relevant and can be safely neglected. The marginal number distribution of the full atomistic simulation, which the AdResS simulation should reproduce, is

$p_{\mathrm{AT}}\left(N_{1}\right)=\frac{Q_{\mathrm{AT}}\left(N_{1}, V_{1}, T\right) Q_{\mathrm{AT}}\left(N-N_{1}, V_{3}, T\right)}{\sum_{n_{1}} Q_{\mathrm{AT}}\left(n_{1}, V_{1}, T\right) Q_{\mathrm{AT}}\left(N-n_{1}, V_{3}, T\right)}$.

We want to calculate the difference between $p\left(N_{1}\right)$ and $p_{\mathrm{AT}}\left(N_{1}\right)$, that is, the difference between the particle probability in the atomistic region of AdResS $\left[p\left(N_{1}\right)\right]$ and the particle probability in a full atomistic simulation in the region equivalent to the AT region of AdResS. In order to do so, we proceed with the following technical operation: We multiply the numerator of $p\left(N_{1}\right)$ by the denominator of $p_{\mathrm{AT}}\left(N_{1}\right)$ (denoted by $T_{1}$ ) and then multiply the numerator of $p_{\mathrm{AT}}\left(N_{1}\right)$ by the denominator of $p\left(N_{1}\right)$ (denoted by $\left.T_{2}\right)$. It follows that

$$
\begin{aligned}
T_{1}= & e^{-\beta N_{1} \omega_{0}} Q_{\mathrm{AT}}\left(N_{1}, V_{1}, T\right) Q_{\mathrm{CG}}\left(N-N_{1}, V_{3}, T\right) \\
& \times \sum_{n_{1}} Q_{\mathrm{AT}}\left(n_{1}, V_{1}, T\right) Q_{\mathrm{AT}}\left(N-n_{1}, V_{3}, T\right), \\
T_{2}= & Q_{\mathrm{AT}}\left(N_{1}, V_{1}, T\right) Q_{\mathrm{AT}}\left(N-N_{1}, V_{3}, T\right) \\
& \times \sum_{n_{1}} e^{-\beta n_{1} \omega_{0}} Q_{\mathrm{AT}}\left(n_{1}, V_{1}, T\right) Q_{\mathrm{CG}}\left(N-n_{1}, V_{3}, T\right) .
\end{aligned}
$$

The difference between $T_{1}$ and $T_{2}$ is basically the difference between $p\left(N_{1}\right)$ and $p_{\mathrm{AT}}\left(N_{1}\right)$. Calculating $T_{1}$,

$$
\begin{aligned}
T_{1}= & \sum_{n_{1}} e^{-\beta N_{1} \omega_{0}} Q_{\mathrm{AT}}\left(n_{1}, V_{1}, T\right) Q_{\mathrm{AT}}\left(N_{1}, V_{1}, T\right) \\
& \times Q_{\mathrm{AT}}\left(N-n_{1}, V_{3}, T\right) Q_{\mathrm{CG}}\left(N-N_{1}, V_{3}, T\right) \\
= & \sum_{n_{1}} \exp \left\{-\beta\left[\omega_{0} N_{1}+A_{\mathrm{AT}}\left(n_{1}, V_{1}, T\right)\right.\right. \\
& +A_{\mathrm{AT}}\left(N_{1}, V_{1}, T\right)+A_{\mathrm{AT}}\left(N-n_{1}, V_{3}, T\right) \\
& \left.\left.+A_{\mathrm{CG}}\left(N-N_{1}, V_{3}, T\right)\right]\right\} .
\end{aligned}
$$


Notice that the free energy is extensive. By applying Euler's theorem [23], we thus find that

$$
\begin{aligned}
A_{\mathrm{AT}}\left(N-n_{1}, V_{3}, T\right) & =V_{3} A_{\mathrm{AT}}\left(\frac{N-n_{1}}{V_{3}}, 1, T\right) \\
& =V_{3} A_{\mathrm{AT}}\left(\rho_{0}+\frac{\hat{N}_{1}-n_{1}}{V_{3}}, 1, T\right), \\
A_{\mathrm{CG}}\left(N-N_{1}, V_{3}, T\right)= & V_{3} A_{\mathrm{CG}}\left(\frac{N-N_{1}}{V_{3}}, 1, T\right) \\
& =V_{3} A_{\mathrm{CG}}\left(\rho_{0}+\frac{\hat{N}_{1}-N_{1}}{V_{3}}, 1, T\right),
\end{aligned}
$$

where $\hat{N}_{1}$ is the number of molecules in the atomistic region at the wished density $\rho_{0}$ of equilibrium, namely, $\hat{N}_{1}=\rho_{0} V_{1}=N-\rho_{0} V_{3}$. As we work under the hypothesis that the atomistic region is much smaller than the whole system, it is reasonable to assume that $N_{1} \ll N$. Moreover, we have found that, in the thermodynamic limit, $N_{1} \sim$ $\hat{N}_{1} \sim \bar{N}_{1}$, which carries over to the running index $n_{1}$ in the grand-canonical partition function that is nonnegligible only if $n_{1} \sim \hat{N}_{1} \sim \bar{N}_{1}$. Hence, $A_{\mathrm{AT}}\left[\rho_{0}+\left(\hat{N}_{1}-\right.\right.$ $\left.\left.n_{1}\right) / V_{3}, 1, T\right]$ and $A_{\mathrm{CG}}\left[\rho_{0}+\left(\hat{N}_{1}-N_{1}\right) / V_{3}, 1, T\right]$ can be regarded as the free energies per unit volume, with the density perturbed from $\rho_{0}$ by $\left(\hat{N}_{1}-n_{1}\right) / V_{3}$ and $\left(\hat{N}_{1}-N_{1}\right) / V_{3}$, respectively. These perturbations originate from the fluctuating number of molecules in the atomistic region: When the number deviates from $\hat{N}_{1}$, molecules enter into the coarse-grained region, and the density is perturbed. In the thermodynamic limit, $\hat{N}_{1}$ is comparable to $n_{1}$ and $N_{1}$, and all of them are supposed to be much smaller than $N$, while $V_{3}$ is of the same order of magnitude of $V$ (the large CG region compared to the small AT region). As a consequence, the perturbations $\left(\hat{N}_{1}-n_{1}\right) /$ $V_{3}$ and $\left(\hat{N}_{1}-N_{1}\right) / V_{3}$ are small, compared to $\rho_{0}=N / V$, and Taylor expanding the free energies (A6) and (A7) about $\rho_{0}$ yields

$$
\begin{aligned}
A_{\mathrm{AT}}\left(\rho_{0}+\frac{\hat{N}_{1}-n_{1}}{V_{3}}, 1, T\right)= & A_{\mathrm{AT}}\left(\rho_{0}, 1, T\right)+\mu_{\mathrm{AT}}\left(\frac{\hat{N}_{1}-n_{1}}{V_{3}}\right) \\
& +\frac{1}{2 \rho_{0}^{2} \kappa_{\mathrm{AT}}}\left(\frac{\hat{N}_{1}-n_{1}}{V_{3}}\right)^{2}+o(1),
\end{aligned}
$$

$$
\begin{aligned}
A_{\mathrm{CG}}\left(\rho_{0}+\frac{\hat{N}_{1}-N_{1}}{V_{3}}, 1, T\right) \\
=A_{\mathrm{CG}}\left(\rho_{0}, 1, T\right)+\mu_{\mathrm{CG}}\left(\frac{\hat{N}_{1}-N_{1}}{V_{3}}\right) \\
\quad+\frac{1}{2 \rho_{0}^{2} \kappa_{\mathrm{CG}}}\left(\frac{\hat{N}_{1}-N_{1}}{V_{3}}\right)^{2}+o(1) .
\end{aligned}
$$

[We use the Landau notation $o(1)$ to collect all terms that are asymptotically negligible, bearing in mind that in the thermodynamic limit $N_{1} \sim \hat{N}_{1}$; see also Ref. [4].] By using the conditions (29) and (30), we have $T_{1}$,

$$
\begin{aligned}
T_{1}= & \sum_{n_{1}} \exp \left\{-\beta\left[A_{\mathrm{AT}}\left(n_{1}, V_{1}, T\right)+A_{\mathrm{AT}}\left(N_{1}, V_{1}, T\right)\right.\right. \\
& +A_{\mathrm{AT}}\left(\rho_{0} V_{3}, V_{3}, T\right)+A_{\mathrm{CG}}\left(\rho_{0} V_{3}, V_{3}, T\right) \\
& +\left(\mu_{\mathrm{AT}}+\mu_{\mathrm{CG}}\right) \hat{N}_{1}-\mu_{\mathrm{AT}}\left(N_{1}+n_{1}\right) \\
& \left.\left.+\frac{V_{3}}{2 \rho_{0} \kappa_{\mathrm{AT}}}\left[\left(\frac{\hat{N}_{1}-n_{1}}{V_{3}}\right)^{2}+\left(\frac{\hat{N}_{1}-N_{1}}{V_{3}}\right)^{2}\right]+o(1)\right]\right\} .
\end{aligned}
$$

Similarly, we calculate $T_{2}$,

$$
\begin{aligned}
T_{2}= & \sum_{n_{1}} e^{-\beta n_{1} \omega_{0}} Q_{\mathrm{AT}}\left(n_{1}, V_{1}, T\right) Q_{\mathrm{AT}}\left(N_{1}, V_{1}, T\right) \\
& \times Q_{\mathrm{CG}}\left(N-n_{1}, V_{3}, T\right) Q_{\mathrm{AT}}\left(N-N_{1}, V_{3}, T\right) \\
= & \sum_{n_{1}} \exp \left\{-\beta\left[\omega_{0} n_{1}+A_{\mathrm{AT}}\left(n_{1}, V_{1}, T\right)+A_{\mathrm{AT}}\left(N_{1}, V_{1}, T\right)\right.\right. \\
& \left.\left.+A_{\mathrm{CG}}\left(N-n_{1}, V_{3}, T\right)+A_{\mathrm{AT}}\left(N-N_{1}, V_{3}, T\right)\right]\right\},
\end{aligned}
$$

with similar expansions, and, by the conditions Eqs. (29) and (30), we have

$$
\begin{aligned}
T_{2}= & \sum_{n_{1}} \exp \left\{-\beta\left[A_{\mathrm{AT}}\left(n_{1}, V_{1}, T\right)+A_{\mathrm{AT}}\left(N_{1}, V_{1}, T\right)\right.\right. \\
& +A_{\mathrm{AT}}\left(\rho_{0} V_{3}, V_{3}, T\right)+A_{\mathrm{CG}}\left(\rho_{0} V_{3}, V_{3}, T\right) \\
& +\left(\mu_{\mathrm{AT}}+\mu_{\mathrm{CG}}\right) \hat{N}_{1}-\mu_{\mathrm{AT}}\left(N_{1}+n_{1}\right) \\
& \left.\left.+\frac{V_{3}}{2 \rho_{0} \kappa_{\mathrm{AT}}}\left[\left(\frac{\hat{N}_{1}-n_{1}}{V_{3}}\right)^{2}+\left(\frac{\hat{N}_{1}-N_{1}}{V_{3}}\right)^{2}\right]+o(1)\right]\right\} .
\end{aligned}
$$

Since the difference between the probabilities $p\left(N_{1}\right)$ and $p_{\mathrm{AT}}\left(N_{1}\right)$ is basically the difference between $T_{1}$ and $T_{2}$ renormalized by the partition functions of the distributions, by invoking Laplace's method again, we see $p\left(N_{1}\right)$ and $p_{\text {AT }}\left(N_{1}\right)$ agree up to the second leading order with respect to the density perturbations $\left(\hat{N}_{1}-n_{1}\right) / V_{3}$ and $\left(\hat{N}_{1}-N_{1}\right) / V_{3}$ in the limit $V_{3} \rightarrow \infty$.

\section{APPENDIX B: SIMULATION PROTOCOLS}

The testing system contains 3456 SPC/E [24] water molecules in a $7.50 \mathrm{~nm} \times 3.72 \mathrm{~nm} \times 3.72 \mathrm{~nm}$ periodic box. The system is divided along the $x$ direction into one atomistic region of width $1.00 \mathrm{~nm}$ and one coarse-grained region of width $1.00 \mathrm{~nm}$ connected by two hybrid regions of width $2.75 \mathrm{~nm}$. The hybrid region is equally divided into three subregions (with a width of approximately $0.9 \mathrm{~nm}$ ) to calculate the local RDFs. These subregions are called HY I, HY II, and HY III, from the AT side to the CG resolution 
side. The simulation is made at room temperature of $300 \mathrm{~K}$. The time step is $\Delta t=0.002 \mathrm{ps}$. The cutoff radius $r_{c}$ used for all interactions is $0.90 \mathrm{~nm}$. The electrostatic interaction method used for the atomistic region is the reaction-field method. All simulations are performed by the MD simulation software GROMACS [21] and VOTCA [25]. In the WCA-based simulation, the interaction potential between particles in the coarse-grained region is given by

$$
U(r)=4 \varepsilon\left[\left(\frac{\sigma}{r}\right)^{12}-\left(\frac{\sigma}{r}\right)^{6}\right]+\varepsilon, \quad r \leq 2^{1 / 6} \sigma .
$$

The parameters used in this study are $\varepsilon=0.65 \mathrm{~kJ} / \mathrm{mol}$ and $\sigma=0.30 \mathrm{~nm}$. These parameters are chosen such that the soft core of WCA is roughly the same as the structurebased coarse-grained water model. Other parameters used in the simulation are the same as in the standard AdResS.

\section{APPENDIX C: THE THIRD-ORDER CORRELATION FUNCTION}

We consider the following definition of the three-body correlation function of a molecular system:

$$
\begin{aligned}
C^{(3)}\left(s_{1}, s_{2}, s_{3}\right)= & \frac{1}{\rho\left(s_{1}\right) \rho\left(s_{2}\right) \rho\left(s_{3}\right)}\left\langle\left[\hat{\rho}\left(s_{1}\right)-\rho\left(s_{1}\right)\right]\right. \\
& \left.\cdot\left[\hat{\rho}\left(s_{2}\right)-\rho\left(s_{2}\right)\right] \cdot\left[\hat{\rho}\left(s_{3}\right)-\rho\left(s_{3}\right)\right]\right\rangle,
\end{aligned}
$$

where the transient density distribution $\hat{\rho}(s)$ and the average density distribution $\rho(s)$ are given by

$$
\hat{\rho}(s)=\sum_{i=1}^{N} \delta\left(s-r_{i}\right) \quad \text { and } \quad \rho(s)=\langle\hat{\rho}(s)\rangle .
$$

By assuming that the system is homogeneous, one easily can show that

$$
\begin{aligned}
C^{(3)}\left(s_{1}, s_{2}, s_{3}\right)= & \frac{1}{\rho^{3}} \rho\left(s_{1}, s_{2}, s_{3}\right)-\frac{1}{\rho^{2}}\left[\rho\left(s_{1}, s_{2}\right)+\rho\left(s_{1}, s_{3}\right)\right. \\
& \left.+\rho\left(s_{2}, s_{3}\right)\right]+2,
\end{aligned}
$$

where

$$
\begin{aligned}
\rho\left(\boldsymbol{s}_{1}, \boldsymbol{s}_{2}\right) & =\left\langle\hat{\rho}\left(\boldsymbol{s}_{1}\right) \hat{\rho}\left(\boldsymbol{s}_{2}\right)\right\rangle \text { and } \\
\rho\left(\boldsymbol{s}_{1}, \boldsymbol{s}_{2}, \boldsymbol{s}_{3}\right) & =\left\langle\hat{\rho}\left(\boldsymbol{s}_{1}\right) \hat{\rho}\left(\boldsymbol{s}_{2}\right) \hat{\rho}\left(\boldsymbol{s}_{3}\right)\right\rangle .
\end{aligned}
$$

Since $g\left(\boldsymbol{s}_{1}, \boldsymbol{s}_{2}\right)=\rho\left(\boldsymbol{s}_{1}, \boldsymbol{s}_{2}\right) / \rho^{2}$ is the two-body radial distribution function, it follows that

$$
\begin{aligned}
C^{(3)}\left(\boldsymbol{s}_{1}, \boldsymbol{s}_{2}, \boldsymbol{s}_{3}\right)= & \frac{1}{\rho^{3}} \rho\left(\boldsymbol{s}_{1}, \boldsymbol{s}_{2}, \boldsymbol{s}_{3}\right) \\
& -\left[g\left(s_{12}\right)+g\left(s_{13}\right)+g\left(s_{23}\right)\right]+2,
\end{aligned}
$$

where, for example, $s_{12}=\left|s_{1}-s_{2}\right|$.

Since we assume the system to be homogeneous, the visualization of this high-dimensional distribution is simplified as follows. We first fix the distance between molecules 1 and 2 (Mol 1 and Mol 2) and then plot the correlation function with respect to the position of the third molecule (Mol 3; see Fig. 9). The position of Mol 3 can be

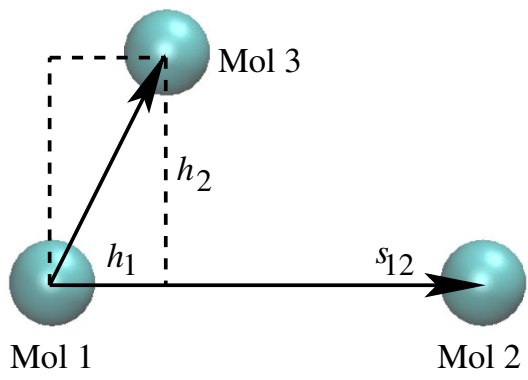

FIG. 9. The schematic plot of the three-body correlation function.

described by the variables $h_{1}$ and $h_{2}$, which are the projection of the position of Mol 3 onto the axis $s_{12}$ defined by Mol 1 and Mol 2.

\section{APPENDIX D: ESTIMATE OF THE EXCESS CHEMICAL POTENTIAL BY AdResS}

In this paper, we have proven that the work of a thermodynamic force and the work of the thermostat in the transition region, which are the ingredients needed to provide density equilibrium across the system, correspond to the difference of the total (kinetic and excess) chemical potential between the atomistic and reservoir regions. Thus, in principle, the calculation of the total work done by the thermodynamic force and that of the thermostat would allow for the determination of, at least, the differences of the total chemical potential, and, when the kinetic part is properly removed, of the differences in the excess chemical potential. While the calculation of the work of the thermodynamic force $\omega_{\text {th }}$ implies simply the straightforward calculation of the integral of such a force over the transition region, the work of the thermostat $\omega_{Q}$, for allowing the proper change of resolution of the molecules across the transition region, is somewhat a much more delicate issue. In essence, the calculation of the chemical potential would require the accurate determination of $\omega_{Q}^{\text {extra }}$, which unfortunately cannot be done in a direct and efficient way. However, we have derived a procedure by which such a quantity can be calculated in an indirect way without additional computational efforts in a standard AdResS simulation. In the following part, we illustrate such a procedure.

\section{Auxiliary Hamiltonian approach}

We define a system that is characterized by an atomistic region, a transition region, and a coarse-grained (reservoir) region, as in a standard AdResS. We couple the different resolutions of the system via a Hamiltonian where the total potential is given by the interpolation of atomistic and coarse-grained potentials in the same fashion of the force interpolation of a standard AdResS:

$$
U=\sum_{i<j} w_{i} w_{j} U_{i j}^{\mathrm{AT}}+\sum_{i<j}\left(1-w_{i} w_{j}\right) U_{i j}^{\mathrm{CG}} .
$$


Next, we determine the thermodynamic force for this system, which leads to the same equilibrium density as that of the standard AdResS. We indicate such a force as $\boldsymbol{F}_{\text {th }}^{H}$, and the superscript $H$ is used to distinguish it from the thermodynamic force that is calculated with the standard AdResS, which we have indicated as $\boldsymbol{F}_{\text {th }}$. We now have the following situation: In the approach based on the Hamiltonian, the change of resolution in the transition region at equilibrium is provided by the work of $\boldsymbol{F}_{\text {th }}^{H}$ (here indicated as $\omega_{\mathrm{th}}^{H}$ ) and by the work of the thermostat to thermalize the reintroduced or removed degrees of freedom $\omega_{\text {DOF }}$. Instead, in the standard AdResS, we have the same equilibrium density of the Hamiltonian approach, but the change of resolution is provided by $\boldsymbol{F}_{\mathrm{th}}, \omega_{\mathrm{DOF}}$, and $\omega_{Q}^{\text {extra }}$. Since we have the same equilibrium, and $\omega_{\text {DOF }}$ is the same in both cases, it follows that

$$
\omega_{Q}^{\text {extra }}=\omega_{\text {th }}^{H}-\omega_{\text {th }}
$$

The limitation of this procedure would be the fact that $\omega_{Q}^{\text {extra }}$ (and thus the chemical potential) could not be calculated within a standard AdResS simulation, and one would require an auxiliary simulation, although the calculation is not computationally demanding. However, based on an extended number of numerical tests, we could develop this idea further and calculate it by the standard AdResS simulation. To do so, we have numerically studied the quantity $\Delta \boldsymbol{F}_{\text {th }}=\boldsymbol{F}_{\text {th }}^{H}-\boldsymbol{F}_{\text {th }}$ for an extended number of systems and proven that such a quantity is equal to $\left\langle w \nabla w\left(U^{\mathrm{AT}}-U^{\mathrm{CG}}\right)\right\rangle$. (In Fig. 10, the case of liquid water

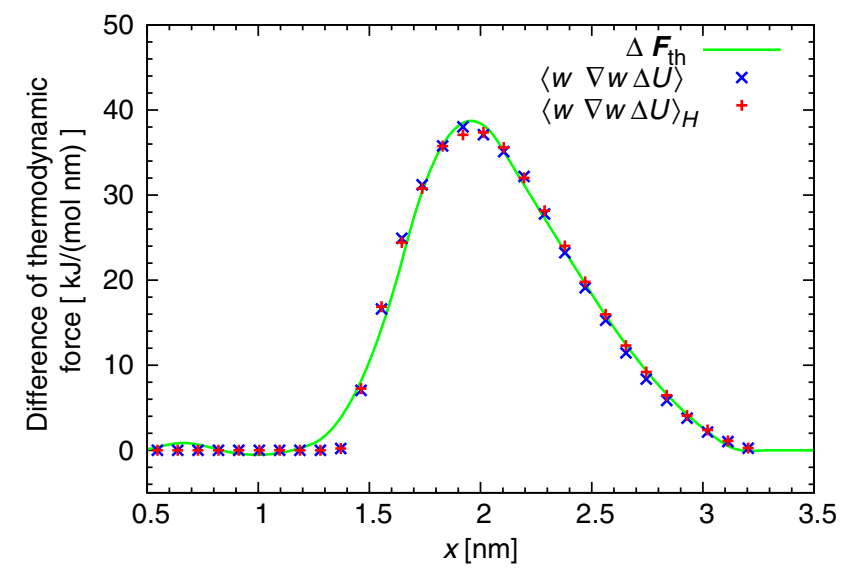

FIG. 10. The difference between the thermodynamic force of the potential interpolation-based scheme and the force interpolation of the standard AdResS. The green solid line shows the difference of the thermodynamic forces, i.e., $\Delta \boldsymbol{F}_{\mathrm{th}}=\boldsymbol{F}_{\mathrm{th}}^{H}-\boldsymbol{F}_{\mathrm{th}}$. The blue and red symbols indicate the quantity $\left\langle w \nabla w\left(U^{\mathrm{AT}}-\right.\right.$ $\left.\left.U^{\mathrm{CG}}\right)\right\rangle$ calculated in the two different approaches. (Blue corresponds to the standard AdResS, while red corresponds to the Hamiltonian-based approach.) The equivalence between the two approaches is suggested by the fact that $\Delta \boldsymbol{F}_{\text {th }}$ corresponds to $\left\langle w \nabla w\left(U^{\mathrm{AT}}-U^{\mathrm{CG}}\right)\right\rangle$ and that such a term is actually the same, independently from the approach used to calculate it. is reported.) This quantity can easily be calculated without additional costs in a standard AdResS simulation, so $\omega_{Q}^{\text {extra }}$ is calculated by

$$
\omega_{Q}^{\mathrm{extra}}=\int d \boldsymbol{r} \Delta \boldsymbol{F}_{\mathrm{th}}=\int d \boldsymbol{r}\left\langle w \nabla w\left(U^{\mathrm{AT}}-U^{\mathrm{CG}}\right)\right\rangle .
$$

The quantity $-\left\langle w \nabla w\left(U^{\mathrm{AT}}-U^{\mathrm{CG}}\right)\right\rangle$ is the average of the additional force (with respect to the standard AdResS) one would have if the intermolecular force is calculated from Eq. (D1) as it happens in the Hamiltonian approach. Such a result provides some physical arguments for the equality with $\Delta \boldsymbol{F}_{\text {th }}$ (besides the numerical evidence employed by us in the first instance). In fact, if one writes down the average total force that acts in the Hamiltonian approach and that acts in the standard AdResS, the difference between them is $\boldsymbol{F}_{\mathrm{th}}-\boldsymbol{F}_{\mathrm{th}}^{H}+\left\langle w \nabla w\left(U^{\mathrm{AT}}-U^{\mathrm{CG}}\right)\right\rangle$. Since $\Delta \boldsymbol{F}_{\mathrm{th}}=$ $\left\langle w \nabla w\left(U^{\mathrm{AT}}-U^{\mathrm{CG}}\right)\right\rangle$, it follows that the total average force that acts in the two approaches is the same, which further implies that the two approaches are essentially equivalent from a numerical point of view, as one would expect, since they have the same equilibrium. However, this procedure is based mainly on practical considerations and numerical experiments; thus, at this stage, it must be considered by the reader as a preliminary procedure, although the essential ingredients already seem to be well defined. Instead, an elegant procedure for an adaptive simulation based on the interpolation of potentials and rigorous thermodynamics considerations has recently been presented by Potestio et al. [26]. Finally, as already stated before in this work, the approach based on the Hamiltonian shall not be considered a proper Hamiltonian approach to adaptive resolution but only an auxiliary tool to determine in a simple way a specific quantity of interest. In fact, if the approach mentioned above is considered a proper Hamiltonian approach, one would have the physical inconsistency that is underlined in Ref. [12], that is, the inevitable fact that the total potential (i.e., the interpolated potential plus the potential corresponding to the thermodynamic force) in either the coarse-grained region or the atomistic region is not that of the original system (as it should be in a proper adaptive Hamiltonian procedure). However, in our view, the most important limitation is that, as is shown in the current work, in this case, the numerical accuracy can be assured only at the first order, and there would not be a systematic way to improve it. The accuracy only at the first order is the difference with the force interpolation-based method proposed here, where corrective forces in the transition region impose numerical correctness of the probability distribution of the system at higher orders. At this point, according to the procedure presented above, we have all the ingredients to calculate the excess chemical potential using AdResS and to compare it with the same quantity that is calculated with the IPM. The calculation is done in the next section. 


\section{Calculation of $\boldsymbol{\mu}_{\mathrm{exc}}$ and numerical experiments}

For the difference of the total chemical potential between the coarse-grained and atomistic regions, we have

$$
\mu^{\mathrm{AT}}-\mu^{\mathrm{CG}}=\int \boldsymbol{F}_{\mathrm{th}} d x+\omega_{Q}^{\mathrm{extra}}+\omega_{\mathrm{DOF}}
$$

The excess chemical potential is defined by removing the kinetic contribution from the total chemical potential, i.e., $\mu_{\text {exc }}=\mu-\mu_{\text {kin }}$. ( $\mu_{\text {kin }}$ is the kinetic part of the chemical potential, which can be calculated analytically.) It follows that $\mu_{\mathrm{exc}}^{\mathrm{AT}}-\mu_{\mathrm{exc}}^{\mathrm{CG}}=\int \boldsymbol{F}_{\mathrm{th}} d x+\omega_{Q}^{\mathrm{extra}}+\omega_{\mathrm{DOF}}-\Delta \mu_{\mathrm{kin}}=$ $\int\left[\boldsymbol{F}_{\mathrm{th}}+\left\langle w \nabla w\left(U^{\mathrm{AT}}-U^{\mathrm{CG}}\right)\right\rangle\right] d x+\omega_{\mathrm{DOF}}-\Delta \mu_{\text {kin }}$. Next, by knowing $\mu_{\mathrm{exc}}^{\mathrm{CG}}$, which can be calculated at rather low cost with the IPM, one obtains $\mu_{\mathrm{exc}}^{\mathrm{AT}}$, whose calculation is instead very expensive with the IPM.

We have carried out several numerical tests interfacing spherical systems with different representations; we have always found a very satisfactory agreement between the chemical potential calculated with AdResS and that calculated with the IPM. More importantly, we have applied this idea to liquid water and found a value of $-22.8 \mathrm{~kJ} / \mathrm{mol}$ with AdResS and a value of $-24.6 \mathrm{~kJ} / \mathrm{mol}$ with the IPM (still not fully converged after about $400 \mathrm{~ns}$ ), which must be compared with the value of $-23.5 \mathrm{~kJ} / \mathrm{mol}$ from the literature [11]. The difference between the value from our approach and that from the IPM is about $8 \%$, which is not significant, but the computational costs of the IPM are much larger than those based on the use of AdResS.

[1] M. Praprotnik, L. Delle Site, and K. Kremer, Adaptive Resolution Molecular-Dynamics Simulation: Changing the Degrees of Freedom on the Fly, J. Chem. Phys. 123, 224106 (2005).

[2] M. Praprotnik, L. Delle Site, and K. Kremer, Adaptive Resolution Scheme for Efficient Hybrid AtomisticMesoscale Molecular Dynamics Simulations of Dense Liquids, Phys. Rev. E 73, 066701 (2006).

[3] S. Fritsch, S. Poblete, C. Junghans, G. Ciccotti, L. Delle Site, and K. Kremer, Adaptive Resolution Molecular Dynamics Simulation through Coupling to an Internal Particle Reservoir, Phys. Rev. Lett. 108, 170602 (2012).

[4] H. Wang, C. Schütte, and L. Delle Site, Adaptive Resolution Simulation (AdResS): A Smooth Thermodynamic and Structural Transition from Atomistic to Coarse Grained Resolution and Vice Versa in a Grand Canonical Fashion, J. Chem. Theory Comput. 8, 2878 (2012).

[5] J.D. Weeks, D. Chandler, and H.C. Anderson, Role of Repulsive Forces in Determining the Equilibrium Structure of Simple Liquids, J. Chem. Phys. 54, 5237 (1971).

[6] T. Çagin and B. M. Pettitt, Grand Molecular Dynamics: A Method for Open Systems, Mol. Simul. 6, 5 (1991).

[7] T. Çăgin and B. M. Pettitt, Molecular Dynamics with a Variable Number of Molecules, Mol. Phys. 72, 169 (1991).

[8] J. Ji, T. Çagin, and B. M. Pettitt, Dynamic Simulations of Water at Constant Chemical Potential, J. Chem. Phys. 96, 1333 (1992).
[9] S. Weerasinghe and B. M. Pettitt, Ideal Chemical Potential Contribution in Molecular Dynamics Simulations of the Grand Canonical Ensemble, Mol. Phys. 82, 897 (1994).

[10] G. C. Lynch and B. M. Pettitt, Grand Canonical Ensemble Molecular Dynamics Simulations: Reformulation of Extended System Dynamics Approaches, J. Chem. Phys. 107, 8594 (1997).

[11] H. Eslami and F. Müller-Plathe, Molecular Dynamics Simulation in the Grand Canonical Ensemble, J. Comput. Chem. 28, 1763 (2007).

[12] L. Delle Site, Some Fundamental Problems for an EnergyConserving Adaptive-Resolution Molecular Dynamics Scheme, Phys. Rev. E 76, 047701 (2007).

[13] M. Praprotnik, S. Poblete, L. Delle Site, and K. Kremer, Comment on "Adaptive Multiscale Molecular Dynamics of Macromolecular Fluids,” Phys. Rev. Lett. 107, 099801 (2011).

[14] O. Lanford, in Statistical Mechanics and Mathematical Problems, Lecture Notes in Physics Vol. 20 (SpringerVerlag, Berlin, 1973), p. 1-113.

[15] D. Ruelle, Superstable Interactions in Classical Statistical Mechanics, Commun. Math. Phys. 18, 127 (1970).

[16] A. Dembo and O. Zeitouni, Large Deviations: Techniques and Applications (Springer-Verlag, Berlin, 2010).

[17] S. Poblete, M. Praprotnik, K. Kremer, and L. Delle Site, Coupling Different Levels of Resolution in Molecular Simulations, J. Chem. Phys. 132, 114101 (2010).

[18] D. Mukherji, N. F. A. van der Vegt, K. Kremer, and L. Delle Site, Kirkwood-Buff Analysis of Liquid Mixtures in an Open Boundary Simulation, J. Chem. Theory Comput. 8, 375 (2012).

[19] H. Wang, C. Junghans, and K. Kremer, Comparative Atomistic and Coarse-Grained Study of Water: What Do We Lose by Coarse-Graining?, Eur. Phys. J. E 28, 221 (2009).

[20] B. Widom, Some Topics in the Theory of Fluids, J. Chem. Phys. 39, 2808 (1963).

[21] B. Hess, C. Kutzner, D. van der Spoel, and E. Lindahl, GROMACS 4: Algorithms for Highly Efficient, LoadBalanced, and Scalable Molecular Simulation, J. Chem. Theory Comput. 4, 435 (2008).

[22] R. Delgado-Buscalioni, K. Kremer, and M. Praprotnik, Coupling Atomistic and Continuum Hydrodynamics through a Mesoscopic Model: Application to Liquid Water, J. Chem. Phys. 131, 244107 (2009).

[23] M.E. Tuckerman, Statistical Mechanics: Theory and Molecular Simulation (Oxford University Press, New York, 2010).

[24] H. J. C. Berendsen, J. R. Grigera, and T. P. Straatsma, The Missing Term in Effective Pair Potentials, J. Phys. Chem. 91, 6269 (1987).

[25] V. Rühle, C. Junghans, A. Lukyanov, K. Kremer, and D. Andrienko, Versatile Object-Oriented Toolkit for CoarseGraining Applications, J. Chem. Theory Comput. 5, 3211 (2009).

[26] R. Potestio, S. Fritsch, P. Espanol, R. Delgado-Buscalioni, K. Kremer, R. Everaers, and D. Donadio, Hamiltonian Adaptive Resolution Simulation for Molecular Liquids, arXiv:1212.4749v1 [Phys. Rev. Lett. (to be published)]. 